\title{
The Chow Motive of the Godeaux surface
}

\author{
V. Guletskiǐ * \\ Institute of Mathematics, \\ Surganova 11, Minsk 220072, Belarus \\ e-mail: guletskii@im.bas-net.by \\ C. Pedrini * \\ Dipartimento di Matematica, Università di Genova, \\ Via Dodecaneso 3516146 - Genova, Italy \\ e-mail: pedrini@dima.unige.it
}

June 7, 2001

\begin{abstract}
Let $X$ be the Godeaux surface obtained as a quotient of the Fermat quintic in $\mathbb{P}_{\mathbb{C}}^{3}$ under the appropriate action of $\mathbb{Z} / 5$. We show that its Chow motive $h(X)$ splits as $1 \oplus 9 \mathbb{L} \oplus \mathbb{L}^{2}$ where $\mathbb{L}=\left(\mathbb{P}^{1},\left[\mathbb{P}^{1} \times p t\right]\right)$ is the Lefschetz motive. This provides a purely motivic proof of the Bloch conjecture for $X$. Our results also give a motivic proof of the Bloch conjecture for those surfaces considered in $[\mathrm{BKL}]$, i.e. all surfaces with $p_{g}=0$ which are not of general type.
\end{abstract}

\section{Introduction}

Let $\mathcal{V}$ be the category of smooth projective varieties over a field $k$. For a variety $X \in \mathrm{Ob}(\mathcal{V})$ let $A^{i}(X)=C H^{i}(X) \otimes \mathbb{Q}$ be the Chow-group of codimension $i$ cycles with $\mathbb{Q}$-coefficients on $X$. For any $X, Y \in \operatorname{Ob}(\mathcal{V})$ the group $A^{0}(X, Y)$ of correspondences (of degree zero) from $X$ into $Y$ is, by definition, the direct sum $\oplus_{j} A^{d_{j}}\left(X_{j} \times Y\right)$ where $X_{j}$ are the irreducible components of $X$ and $d_{j}=\operatorname{dim}\left(X_{j}\right)$ for any $j$. If $f \in A^{0}(X, Y)$ and $g \in A^{0}(Y, Z)$ for three varieties $X, Y$ and $Z$ then the composition $g \circ f \in A^{0}(X, Z)$ can be defined by the usual formula $g \circ f=p_{X Z_{*}}\left(p_{X Y}^{*}(f) \cdot p_{Y Z}^{*}(g)\right)$ where $p_{X Z}, p_{X Y}$ and $p_{Y Z}$ are the appropriate projections. Varieties and correspondences form an additive category. Its pseudoabelian envelope $\mathcal{M}$ is called the category of Chow motives over $k$ with coefficients in $\mathbb{Q}$. Objects in $\mathcal{M}$ are pairs $(X, p)$ where $X \in \mathrm{Ob}(\mathcal{V})$ and $p$ is a projector of $X$, that is a correspondence $p \in A^{0}(X, X)$, such that $p \circ p=p$. For any two motives $M=(X, p)$ and $N=(Y, q)$ a morphism $f: M \rightarrow N$ is a correspondence $f \in A^{0}(X, Y)$, such that $q \circ f=f=f \circ p$. Since the category $\mathcal{M}$ is pseudo-abelian, every projector on $M \in \mathrm{Ob}(\mathcal{M})$ induces a decomposition of $M$ as the sum of $\operatorname{ker}(p)$ and $\operatorname{im}(p)$. If $f \in A^{*}(X \times Y)$ by $f^{t} \in A^{*}(Y \times X)$ we denote the transpose of $f$.

The diagonal $\Delta_{X}$ of a variety $X$ is a projector of $X$ and $h(X)=\left(X, \Delta_{X}\right)$ is called the motive of the variety $X$. If $M=(X, p)$ is a motive then its identity morphism $1_{M}: M \rightarrow M$ is given by

${ }^{*}$ The authors acknowledge the support of TMR ERB FMRX CT-97-0107. The first named author also acknowledges SFB 343 "Diskrete Strukturen in der Mathematik" and the hospitality of the University of Bielefeld. The second named author is a member of GNSAGA of CNR. 
the projector $p$. In particular, $\Delta_{X}$ can be viewed as the identity morphism of $h(X)$ for any variety $X$. If $f: Y \rightarrow X$ is a morphism of varieties then its graph $\Gamma_{f}: h(X) \rightarrow h(Y)$ is a morphism of its Chow motives: hence $X \rightarrow h(X), f \rightarrow \Gamma_{f}$ is a contravariant functor from the category of varieties $\mathcal{V}$ into the category of Chow motives $\mathcal{M}$. The Chow groups of a motive $M$ are defined by $A^{i}(M)=p_{*}\left(A^{i}(X)\right)$ (see [Mu1]).

By $1=h(\operatorname{Spec}(k))$ we will denote the unit motive and by $\mathbb{L}=\left(\mathbb{P}_{k}^{1},\left[\mathbb{P}_{k}^{1} \times p\right]\right)$, where $p$ is a rational point of $X$, the Lefschetz motive. $\mathbb{L}$ can also be defined by the equality: $h\left(\mathbb{P}^{1}\right)=1 \oplus \mathbb{L}$ (see [Ful]). In general: if $X$ is irreducible of dimension $d$ and $p$ is a rational point on $X$ then one has: $(X,[p \times X]) \cong 1$ and $(X,[X \times p]) \cong \mathbb{L}^{d}$, where $\mathbb{L}^{d}$ is the $d$-fold tensor product of the motive $\mathbb{L}$ by itself (see Section 3$)$.

Now let $k$ be an algebraically closed field of characteristic zero and let $H$ be a Weil cohomology theory on the category of smooth projective varieties over $k$. Then for every variey $X$ there exists a graded algebra $H^{*}(X)$ and the usual axioms hold (see [Kl]). In particular there is a cycle map $c l_{X}^{i}: A^{i}(X) \rightarrow H^{2 i}(X)$. For example, if $k=\mathbb{C}$ one can take for $H^{*}(X)$ the singular cohomology groups $H^{*}(X, \mathbb{Q})$ of the underlying manifold $X(\mathbb{C})$ with coefficients in $\mathbb{Q}$.

The group of codimension $i$ cycles homologically equivalent to 0 is then defined to be $A_{\text {hom }}^{i}(X)=$ $\left\{\alpha \in A^{i}(X) \mid c l_{X}^{i}(\alpha)=0\right\}$. For every motive $M=(X, p)$ its cohomology groups are defined by $H^{i}(M)=p_{*}\left(H^{i}(X)\right)$. As the cycle maps of varieties commute with correspondences one can similarly define the cycle class of a motive. By $b_{i}(X)$ we will denote the Betti number $\operatorname{dim}\left(H^{i}(X, \mathbb{Q})\right)$ of a complex variety $X$.

One of the crucial questions that arise in this context is to ask how much of the properties of the Chow graded ring $A(X)=\oplus A^{i}(X)$ can be recovered by looking at the Chow motive $h(X)$. In particular Beilinson conjectured the existence of a filtation $\left\{F^{*}\right\}$ on $A(X)$ (see [Ja], 22 ), such that $F^{0} A^{j}(X)=A^{j}(X), F^{1} A^{j}(X)=A^{j}(X)_{h o m}$ and the associated graded groups $G r_{F}^{\nu} A^{j}(X)=$ $F^{\nu} A^{j}(X) / F^{\nu+1} A^{j}(X)$ depend only on the motive of $X$ modulo homological equivalence (i.e. the correspondences in $A^{i}(X \times X)_{h o m}$ act as 0 on $\left.G r_{F}^{\nu} A^{j}(X)\right)$.

In this paper we will consider the case of a particular surface $X$ over $\mathbb{C}$ of general type with $p_{g}=0$, the so called classical Godeax surface. We prove (Theorem 4.3) that the motive $h(X)$ is isomorphic to the direct sum of "trivial" motives in the sense that $h(X) \cong 1 \oplus 9 \mathbb{L} \oplus \mathbb{L}^{2}$, where $9=b_{2}(X)$ and $9 \mathbb{L}$ is a direct sum of 9 copies of $\mathbb{L}$. This shows that $h(X)$ is isomorphic to the motive of a rational surface having the same Betti numbers as $X$. Therefore Theorem 4.3 appears as a natural extension of a known result for Enriques surfaces: if $X$ is an Enriques surface then $h(X)$ is isomorphic to the motive of a rational surface $J$, [Co]. In this case $J$ is the Jacobian fibration associated to the elliptic fibration $\pi: X \rightarrow \mathbb{P}^{1}$.

From this one immediately deduce the so called Bloch Conjecture for the Godeaux surface $X$ because $A^{2}(X) \cong A\left(\mathbb{L}^{2}\right)=\mathbb{Q}$.

The computation of $A^{2}(X)$ for the Godeaux surface has been done in different ways by Inose and Mizukami ([InMiz]) and by C. Voisin ([Voi]), while the problem of proving Bloch's conjecture for all surfaces of general type with $p_{g}=0$ remains still open.

Our motivic proof is along the line of the ideas of Bloch and Beilinson: let $T(X)=\operatorname{ker}\left(A_{0}(X) \rightarrow\right.$ $\operatorname{Alb}(X))$ be the Albanese kernel of $X$, where $A_{0}(X)$ denotes the group of 0 -cycles of degree 0 . Then, if a Beilinson's filtration $\left\{F^{*}\right\}$ exists for a smooth projective variety $X$ of dimension $d$, we must have $F^{2} A^{d}(X)=T(X)([\mathrm{Ja}], 2.10)$.

Bloch ([Bl], 1.11) proves that if $X$ is a surface with $p_{g}=0$, such that the action of correspondences on $T(X)$ factors trough homological equivalence, then $T(X)$ is 0 .

We show that the motive of the Godeaux surface $X$ is finite dimensional in the sense of 
S. Kimura (see $[\mathrm{Ki}]$ ). This implies that if a correspondence acts as 0 on the cohomology of $X$ then it is a nilpotent as a morphism of $h(X)$.

More generally we prove (Theorem 3.18) that, if $X$ is a complex surface such that $h(X)$ is finite dimensional and $p_{g}(X)=0$, then $T(X)=0$. This result yields (Corollary 3.19) a motivic proof of the Bloch conjecture for all surfaces with $p_{g}=0$ which are not of general type.

We can compare our result with a theorem of J. Murre on the so called Chow-Kunneth decomposition of a surface $X$. In general Murre [Mu2] conjectured that for a smooth projective variety $X$ of dimension $d$ there exists a decomposition of the motive $h(X)$ :

$$
h(X)=\bigoplus_{0 \leq i \leq 2 d} h^{i}(X)
$$

where $h^{i}(X)=\left(X, \pi_{i}\right), \pi_{i}$ are pairwise orthogonal projectors, such that $\operatorname{cl}\left(\pi_{i}\right)$ coincides with the $(2 d-i, i)$-component of $\Delta_{X}$ in the Kunneth decompositon of $H^{2 d}(X \times X, \mathbb{Q})$ and $\pi_{0}, \ldots, \pi_{j-1}$ and $\pi_{2 j+1}, \ldots, \pi_{2 d}$ act trivially on $A^{j}(X)$. Also $h^{0}(X)=\left(X, \pi_{0}\right) \cong 1$ and $h^{2 d}(X)=\left(X, \pi_{2 d}\right) \cong \mathbb{L}^{d}$.

By setting $F^{0} A^{j}(X)=A^{j}(X)$ and $F^{k} A^{j}(X)=\operatorname{ker}\left(\pi_{2 j+1-k} \mid F^{k-1}\right)$ one inductively defines a filtration on $A^{*}(X)$. Jannsen proved in [Ja], $\S 5$, that the existence of a Chow-Kunnet decomposition with $F^{1} A^{j}(X)=A^{j}(X)_{h o m}$ is, in fact, equivalent to the existence of a Beilinson filtration on $A^{*}(X)$.

Murre [Mu1] proved that such a decomposition exists when $\operatorname{dim}(X)=2$. In this case the problem of understanding $h(X)$ reduces to the case of $h^{2}(X)$, a motive which carries at the same time the Neron-Severi group of $X$, the Albanese kernel $T(X)$ and the group of transcendental cycles. If $X=\mathbb{P}^{2}$ then $h^{2}(X)$ is "trivial", i.e. $h^{2}(X) \cong \mathbb{L}$. Here we prove (Th. 3.21) that, if $X$ is a smooth projective surface with $p_{g}(X)=q(X)=0$ and $h(X)$ is finite dimensional, then $h^{2}(X)$ is a direct sum of trivial motives, i.e. $h^{2}(X) \cong b_{2}(X) \cdot \mathbb{L}$.

The paper is organized as follows: in $\S 2$ we recall, following [Re], some properties of the construction of the Godeaux surface $X$ as the quotient of a quintic surface $Y$ in $\mathbb{P}^{3}$ which is fixed under the action of $\mathbb{Z} / 5$ defined by $\left(x_{i}\right) \mapsto\left(\epsilon^{i} x_{i}\right)$ where $\epsilon$ is a primitive 5 -th root of $1 . X$ has an involution $\beta$, such that $F i x(\beta)$ consists of a curve $M_{1} \simeq \mathbb{P}^{1}$ with $M_{1}^{2}=-3$ together with 5 isolated points $Q_{0}, \cdots, Q_{4}$. The quotient $S=X / \beta$ is then a rational surface with 5 nodes. $S$ can also be thought as the quotient of $Y$ under a dihedral group $D_{10}$ generated by $\mathbb{Z} / 5$ and $\beta$. The linear system $\Phi=\left|3 K_{X}+M_{1}\right|$ on $X$ induces a pencil $X \rightarrow \mathbb{P}^{1}$ which has five reducible fibers at the points $Q_{i}$ and a genus 2 curve as a generic fiber. We find (Theorem 2.1) an explicit base for the 9-dimensional vectors space $H^{2}(X, \mathbb{Q})$ consisting of 3 rational curves $M_{1}, M_{2}, M_{3}$, of $K_{X}$ and of 5 elliptic curves each one passing through a point $Q_{i}$. Then we show (Prop. 2.2) that the above basis is invariant under the action of the involution $\beta$ and that $b_{i}(\tilde{X})=b_{i}(\tilde{S})$ where $\tilde{X}$ is the blow up of $X$ at the 5 points $Q_{i}$ and $\tilde{S}$ is a non singular model of $S$.

In $\S 3$ we recall some definitions and results of S. Kimura [Ki] on finite dimensional motives that we will need for the proof of Theorem 4.3. In particular it follows that the class of finite dimensional motives is closed under direct sums (Prop. 3.3), tensor products (Prop. 3.4) and blow-ups (Prop 3.6). We also show (Theorem 3.13) that the finite-dimensionality of the motive $h(X)$ is a birational invariant for a smooth projective surface. It follows that the motive of a rational surface is finite diemnsional. Moreover if a finite dimensional motive $M$ is such that $H^{*}(M)=0$ then $M=0$ (Cor. 3.17). These results in particular imply that if $X$ is a smooth projective surface over $\mathbb{C}$ with $p_{g}=0$ and the Chow motive $M=h(X)$ is finite dimensional, then the Albanese kernel $T(X)$ vanishes (Theorem 3.18). This notably applies (Corollary 3.19) to all complex surfaces with $p_{g}=0$ and Kodaira dimension less than 2. Theorem 3.21 shows that if $p_{g}=q=0$ and the motive $h(X)$ is finite dimensional then $h(X)=1 \oplus b_{2} \cdot \mathbb{L} \oplus \mathbb{L}^{2}$. 
In $\S 4$ we show that the motive $h(X)$ of the Godeaux surface $X$ is finite dimensional. Using this results we compute $h(X)$ (Theorem 4.3).

The proof of our result suggests that some of the above arguments can be extended to other surfaces of general type coming from the action of a dihedral group on a smooth projective surface in $\mathbb{P}^{n}$. For example, a Campedelli surface $X$ (for which $p_{g}=q=0$ and $K_{X}^{2}=2$ ) can be obtained from the action of a dihedral group $D_{14}$ generated by $\mathbb{Z} / 7$ and an involution $\beta$ on a smooth surface $Y \subset \mathbb{P}^{5}$ (see $[\mathrm{Re}]$, p. 323). In this case Fix $(\beta)$ consists of rational curve $m$ with $m^{2}=-4$ together with 6 isolated points. The quotient surface $S=X / \beta$, where $X=Y /(\mathbb{Z} / 7)$, has a branch curve $C$ (the image of $m$ ) of order 10 with $C^{2}=-8$. The minimal model of the pair $S \& C$ (in the sense of Iithaka (see [Re], p. 346)) is $\mathbb{P}^{2}$ with a curve of degree 10 having 6 triple tacnodes. However computations in this case seem much harder: $Y$ has degree 14 and it can be described in terms of pfaffians of a $7 \times 7$ skew symmetric matrix. One should prove that $h(Y)$ is finite dimensional. Then $h(X)$ would be finite dimensional and our results will apply : $h(X)=1 \oplus 8 \cdot \mathbb{L} \oplus \mathbb{L}^{2}$.

The authors wish to thank I. Panin for many helpful conversations over the subject of this paper.

\section{A basis for the cohomology of the Godeaux surface}

We recall a classical construction of the Godeaux surface. Let $\mu_{5}$ be the group of 5 -th roots of unity generated by the primitive root $\epsilon$. Let $\mu_{5}$ act on $\mathbb{P}^{3}$ by the rule

$$
\left(x_{1}: x_{2}: x_{3}: x_{4}\right) \mapsto\left(\epsilon x_{1}: \epsilon^{2} x_{2}: \epsilon^{3} x_{3}: \epsilon^{4} x_{4}\right) .
$$

Let $f=f\left(x_{1}, \ldots, x_{4}\right)$ be a $\mu_{5}$-invariant quintic form. If $f\left(P_{i}\right) \neq 0$ where $P_{i}$ are the four coordinate points $(0, \ldots, 1, \ldots, 0)$ then the quintic surface in $\mathbb{P}^{3}$ defined by $f=0$ is invariant under $\mu_{5}$ and $Y$ is non singular. For the sake of simplicity we will take $Y$ to be the Fermat quintic defined by the equation

$$
x_{1}^{5}+x_{2}^{5}+x_{3}^{5}+x_{4}^{5}=0 .
$$

The quotient $X=Y / \mu_{5}$ is the Godeaux surface. Let $\eta: Y \rightarrow X$ be the quotient map.

Consider the 3 lines in $\mathbb{P}^{3}$ lying on $Y$ :

$$
\begin{aligned}
& L_{1}: x_{1}=-x_{4}, \quad x_{2}=-x_{3} \\
& L_{2}: x_{1}=-x_{3}, \quad x_{2}=-x_{4} \\
& L_{3}: x_{1}=-x_{2}, \quad x_{3}=-x_{4}
\end{aligned}
$$

We have $L_{s} \cdot L_{t}=1$ for $s \neq t$. Since $Y$ is a degree five hypersurface in $\mathbb{P}^{3}$, the canonical invertible sheaf $\omega_{Y}$ is equal to $\mathcal{O}_{Y}(1)$. The canonical class $K_{Y}$ can be cut by a hyperplane, e.g. $x_{1}=0$. Any $L_{s}$ meets this hyperplane transversally. Hence $K_{Y} \cdot L_{s}=1$ and, by the adjunction formula, $L_{s}^{3}=-3$ for any $s \in\{1,2,3\}$. For any $s$, the orbit of the action of $\mu_{5}$ on $L_{s}$ contains five different lines on $Y$. In other words a nontrivial element $\alpha \in \mu_{5}$ shifts any line $L_{s}$ inside a set of lines on $Y$. It follows that $\eta$ maps $L_{s}$ isomorphically into $X$ for any $s$. Therefore, $M_{s}=\eta\left(L_{s}\right) \simeq \mathbb{P}^{1}$. If $s, t \in\{1,2,3\}$ and $s \neq t$, then $L_{t}$ intersects only one line in the orbit $\sum_{\alpha \in \mu_{5}} \alpha\left(L_{s}\right)$, so that

$$
M_{s} \cdot M_{t}=1
$$

for $s \neq t$. 
Since the map $\eta$ is unramified of degree 5 we have: $K_{X}=\frac{1}{5} \eta_{*} K_{Y}$. The class $K_{Y}$ is very ample and the quotient morphism $\eta$ is a surjective finite map. Therefore (see [Ha], p. 25) $K_{X}$ is ample and

$$
M_{s} \cdot K_{X}=1
$$

for $s=1,2,3$. The equality $K_{Y}^{2}=5$ implies $K_{X}^{2}=1$. By the adjunction formula $M_{s}^{2}=-3$ for $s=1,2,3$.

$X$ is a surface of general type with $p_{g}(X)=0$, hence $q(X)=0$. Therefore the cycle map $c l: A^{1}(X) \rightarrow H^{2}(X, \mathbb{Q})$ is an isomorphism. Since the second Betti number $b_{2}(X)=\operatorname{dim} H^{2}(X, \mathbb{Q})$ of $X$ is equal to 9 , we have that $A^{1}(X) \cong H^{2}(X, \mathbb{Q})$ is a 9 -dimensional $\mathbb{Q}$-vector space.

To find an algebraic basis in $H^{2}(X, \mathbb{Q})$ we will extend the system $M_{1}, M_{2}, M_{3}, K_{X}$ to a system of nine divisors with a non-degenerate intersection matrix.

To do this let's consider the linear system $\Phi=\left|3 K_{X}-M_{1}\right|$ on $X$ (see [Re]). $\Phi$ is a dimension one linear system without fixed points. Therefore it induces a pencil

$$
\Phi: X \rightarrow \mathbb{P}^{1} .
$$

This fibration has five reducible fibers and a genus two curve as a general fiber. The line $L: x_{1}=$ $x_{4}, x_{2}=x_{3}$ intersects the quintic $Y$ in five points $\left(1:-\epsilon^{i}:-\epsilon^{i}: 1\right), i \in\{0,1,2,3,4\}$. Let

$$
Q_{i}=\eta\left(1:-\epsilon^{i}:-\epsilon^{i}: 1\right)
$$

be the corresponding points on the surface $X$. For any $i$ there exist two elliptic curves, say $F_{i}^{+}$ and $F_{i}^{-}$, on the surface $X$ such that

$$
\begin{gathered}
F_{i}^{+} \cap F_{i}^{-}=\left\{Q_{i}\right\}, \\
F_{i}^{+} \cdot F_{i}^{-}=1
\end{gathered}
$$

and the divisors $F_{i}^{+}+F_{i}^{-}$form five reducible fibers of the pencil $\Phi: X \rightarrow \mathbb{P}^{1}$ (see [Re], p. 316).

Theorem 2.1 The cohomology classes of the nine divisors

$$
M_{1}, M_{2}, M_{3}, K_{X}, F_{0}^{+}, F_{1}^{+}, F_{2}^{+}, F_{3}^{+}, F_{4}^{+}
$$

form a basis in the $\mathbb{Q}$-vector space $H^{2}(X, \mathbb{Q})$. The cohomology classes of the nine divisors

$$
M_{1}, M_{2}, M_{3}, K_{X}, F_{0}^{-}, F_{1}^{-}, F_{2}^{-}, F_{3}^{-}, F_{4}^{-}
$$

form a basis in $H^{2}(X, \mathbb{Q})$ as well.

Proof. We divide the proof of this theorem into 3 steps. First we find ten $\mu_{5}$-invariant elliptic curves $E_{i}^{ \pm}$on $Y$, such that $E_{i}^{+}$and $E_{i}^{-}$contain the point $\left(1:-\epsilon^{i}:-\epsilon^{i}: 1\right)$. Then we show that the images of $E_{i}^{ \pm}$under $\eta: Y \rightarrow X$ are the reducible fibers $F_{i}^{ \pm}$of the pencil (1). Using this we compute the intersection matrices of the two collections of divisors indicated in the theorem and show that their determinants are non zero. The theorem will follow.

According to the Theorem on page 362 in [Re] there is a $1-1$ correspondence between points

$$
(a: b: c: d ; \sigma: \tau) \in \mathbb{P}^{3} \times \mathbb{P}^{1}
$$

and $\mu_{5}$-invariant elliptic curves $E(a: b: c: d ; \sigma: \tau) \subset \mathbb{P}^{3}$ defined by the five cubic forms

$$
R_{0}: a x_{1}^{2} x_{3}-b x_{1} x_{2}^{2}+c x_{3}^{2} x_{4}-d x_{2} x_{4}^{2}=0
$$




$$
\begin{aligned}
& R_{1}: b \sigma x_{2}^{3}-a \sigma x_{1} x_{2} x_{3}+a \tau x_{1}^{2} x_{4}+c \tau x_{3} x_{4}^{2}=0 \\
& R_{2}: d \tau x_{4}^{3}+b \tau x_{1} x_{2} x_{4}+b \sigma x_{2}^{2} x_{3}-a \sigma x_{1} x_{3}^{2}=0 \\
& R_{3}: a \tau x_{1}^{3}+c \tau x_{1} x_{3} x_{4}+c \sigma x_{2} x_{3}^{2}-d \sigma x_{2}^{2} x_{4}=0 \\
& R_{4}: c \sigma x_{3}^{3}-d \sigma x_{2} x_{3} x_{4}+d \tau x_{1} x_{4}^{2}+b \tau x_{1}^{2} x_{2}=0
\end{aligned}
$$

Substituting the point $\left(1:-\epsilon^{i}:-\epsilon^{i}: 1\right)$ in the above system of cubics we see that a curve $E(a: b: c: d ; \sigma: \tau)$ contains the point $\left(1:-\epsilon^{i}:-\epsilon^{i}: 1\right)$ if and only if the following identities hold:

$$
a+\epsilon^{i} b-\epsilon^{i} c-d=0, \quad \epsilon^{i} \tau b+\sigma \epsilon^{3 i} c+\left(\sigma \epsilon^{2 i}-\tau\right) d=0 .
$$

For any point $(u: v) \in \mathbb{P}^{1}$ let $Z_{(u, v)}$ be the cubic hypersurface in $\mathbb{P}^{3}$ represented by the equation:

$$
Z_{(u, v)}: u\left(x_{1}^{2} x_{3}+x_{4}^{2} x_{2}\right)+v\left(x_{1} x_{2}^{2}+x_{4} x_{3}^{2}\right)=0 .
$$

$Z_{(u, v)}$ is a member of the pencil in $\mathbb{P}^{3}$ generated by the two cubics

$$
x_{1}^{2} x_{3}+x_{4}^{2} x_{2}, \quad x_{1} x_{2}^{2}+x_{4} x_{3}^{2} .
$$

We want to find elliptic curves of type $E(a: b: c: d ; \sigma: \tau)$ which not only contain the point $\left(1:-\epsilon^{i}:-\epsilon^{i}: 1\right)$ but are also contained in some $Z_{(u, v)}$. To do so we choose $Z_{(u, v)}$ such that $\left(1:-\epsilon^{i}:-\epsilon^{i}: 1\right) \in Z_{(u, v)}$. Substituting $\left(1:-\epsilon^{i}:-\epsilon^{i}: 1\right)$ into the equation of $Z_{u: v}$ we obtain $u=\epsilon^{i} v$, whence $\left(1:-\epsilon^{i}:-\epsilon^{i}: 1\right) \in Z_{\left(\epsilon^{i}, 1\right)}$. Regrouping monomials in the equation of $Z_{\left(\epsilon^{i}, 1\right)}$ we see that the hypersurface $Z_{\left(\epsilon^{i}, 1\right)}$ is defined by the equation

$$
\epsilon^{i} x_{1}^{2} x_{3}-(-1) x_{1} x_{2}^{2}+x_{4} x_{3}^{2}-\left(-\epsilon^{i}\right) x_{4}^{2} x_{2}=0 .
$$

Comparing this equation with the equations definining $E\left(\epsilon^{i}:-1: 1:-\epsilon^{i} ; \sigma: \tau\right)$ we see that if $a=\epsilon^{i}, b=-1, c=1$ and $d=-\epsilon^{i}$ then the corresponding elliptic curve lies on the hypersurface $Z_{\left(\epsilon^{i}, 1\right)}$. Moreover, with such values of $a, b, c, d$ the equations (2) hold, hence $E\left(\epsilon^{i}:-1: 1:-\epsilon^{i} ; \sigma\right.$ : $\tau)$ contains the point $\left(1:-\epsilon^{i}:-\epsilon^{i}: 1\right)$.

From (ii) in $[\operatorname{Re}]$, p. 362, it follows that the curve $E\left(\epsilon^{i}:-1: 1:-\epsilon^{i} ; \sigma: \tau\right)$ lies on $Y$ if and only if the quintic form $x_{1}^{5}+x_{2}^{5}+x_{3}^{5}+x_{4}^{5}$ belongs to the $\mathbb{C}$-vectorspace generated by the seven quintic forms

$$
x_{1}^{2} R_{3}, x_{2}^{2} R_{1}, x_{3}^{2} R_{4}, x_{4}^{2} R_{2}, x_{1} x_{4} R_{0}, x_{2} x_{3} R_{0}, x_{3} x_{4} R_{3} .
$$

A simple computation shows that the 2 elliptic curves:

$$
E_{i}^{+}=E\left(\epsilon^{i}:-1: 1:-\epsilon^{i} ; 1+\sqrt{5}: 2 \epsilon^{2 i}\right)
$$

and

$$
E_{i}^{-}=E\left(\epsilon^{i}:-1: 1:-\epsilon^{i} ; 1-\sqrt{5}: 2 \epsilon^{2 i}\right)
$$

lie on the quintic $Y$.

All curves $E_{i}^{ \pm}$are smooth and invariant under the action of $\mu_{5}$ with no fixed points. Therefore, considering the coverings: $\eta: E_{i}^{ \pm} \rightarrow \eta\left(E_{i}^{ \pm}\right)$, by Hurwitz theorem it follows that $\eta\left(E_{i}^{ \pm}\right)$are elliptic curves and that these coverings are isogenies.

Since both curves $E_{i}^{ \pm}$contain the point $\left(1:-\epsilon^{i}:-\epsilon^{i}: 1\right)$, the curves $\eta\left(E_{i}^{ \pm}\right)$contain the point $Q_{i}$.

We claim that the curves $\eta\left(E_{i}^{ \pm}\right)$do not intersect the general fiber of the pencil $\Phi: X \rightarrow \mathbb{P}^{1}$. To prove the claim we compute the intersection numbers $\eta\left(E_{i}^{ \pm}\right) \cdot M_{1}$ and $\eta\left(E_{i}^{ \pm}\right) \cdot K_{X}$. First we show that $E_{i}^{ \pm} \cdot L_{1}=3$ on the quintic $Y$. It will follow that $\eta\left(E_{i}^{ \pm}\right) \cdot M_{1}=3$. 
To do this we substitute the equations of the line $L_{1}$ into the equations of the elliptic curve $E_{i}^{ \pm}=E\left(\epsilon^{i}:-1: 1:-\epsilon^{i} ; \sigma: \tau\right)$ where $\sigma=1 \pm \sqrt{5}$ and $\tau=2 \epsilon^{2 i}$. Passing to the affine space $x_{2} \neq 0$ we obtain the equation

$$
\epsilon^{i} \tau x^{3}+\tau x^{2}-\epsilon^{i} \sigma x+\sigma=0
$$

where $x=x_{1}$. This equation has three distinct roots, hence the line $L_{1}$ intersects the curve $E_{i}^{ \pm}$in three points $\left\{P_{1}, P_{2}, P_{3}\right\}$. The multiplicity of the intersection $E_{i}^{ \pm} \cdot L_{1}$ in any point $P_{r}$ is equal to 1. To see this let $H: x_{4}=-x_{1}$ and $H^{\prime}: x_{3}=-x_{2}$ : then $L_{1}=H \cap H^{\prime}$ and $E_{i}^{ \pm} \cap L_{1} \subset E_{i}^{ \pm} \cap H$. Let us compute the number of points in the intersection $E_{i}^{ \pm} \cap H$. By affinizing on $x_{1}$ we obtain the system

$$
\begin{gathered}
R_{0}: \epsilon^{i} x_{3}+x_{2}^{2}-x_{3}^{2}+\epsilon^{i} x_{2}=0 \\
R_{1}:-\sigma x_{2}^{3}-\epsilon^{i} \sigma x_{2} x_{3}-\epsilon^{i} \tau+\tau x_{3}=0 \\
R_{2}: \epsilon^{i} \tau+\tau x_{2}-\sigma x_{2}^{2} x_{3}-\epsilon^{i} \sigma x_{3}^{2}=0 \\
R_{3}: \epsilon^{i} \tau-\tau x_{3}+\sigma x_{2} x_{3}^{2}-\epsilon^{i} \sigma x_{2}^{2}=0 \\
R_{4}: \sigma x_{3}^{3}-\epsilon^{i} \sigma x_{2} x_{3}-\epsilon^{i} \tau-\tau x_{2}=0
\end{gathered}
$$

which, by linear transformations, reduces to:

$$
\begin{gathered}
R_{0}: \epsilon^{i} x_{3}+x_{2}^{2}-x_{3}^{2}+\epsilon^{i} x_{2}=0 \\
R_{3}: \epsilon^{i} \tau-\tau x_{3}+\sigma x_{2} x_{3}^{2}-\epsilon^{i} \sigma x_{2}^{2}=0 \\
R_{4}: \sigma x_{3}^{3}-\epsilon^{i} \sigma x_{2} x_{3}-\epsilon^{i} \tau-\tau x_{2}=0
\end{gathered}
$$

Now observe that

$$
\epsilon^{i} x_{3}+x_{2}^{2}-x_{3}^{2}+\epsilon^{i} x_{2}=\epsilon^{i}\left(x_{2}+x_{3}\right)+\left(x_{2}-x_{3}\right)\left(x_{2}+x_{3}\right)=\left(x_{2}+x_{3}\right)\left(\epsilon^{i}+x_{2}-x_{3}\right) .
$$

Therefore $\epsilon^{i} x_{3}+x_{2}^{2}-x_{3}^{2}+\epsilon^{i} x_{2}=0$ if and only if either $x_{2}=-x_{3}$ or $x_{3}=x_{2}+\epsilon^{i}$. Let $H^{\prime \prime}: x_{3}=$ $x_{2}+\epsilon^{i}$. Then the set of points in $E_{i}^{ \pm} \cap H$ is a disjoint union of the sets $E_{i}^{ \pm} \cap\left(H \cap H^{\prime}\right)=\left\{P_{1}, P_{2}, P_{3}\right\}$ and $E_{i}^{ \pm} \cap\left(H \cap H^{\prime \prime}\right)$. Substituting $x_{2}+\epsilon^{i}$ to $x_{3}$ in the last system we see that the set $E_{i}^{ \pm} \cap\left(H \cap H^{\prime \prime}\right)$ contains two distinct points $P_{4}$ and $P_{5}$. Therefore $E_{i}^{ \pm} \cap H=\left\{P_{1}, P_{2}, P_{3}, P_{4}, P_{5}\right\}$. Since any elliptic curve $E_{i}^{ \pm}$has degree 5 (see [Re], Theorem on page 362), by Bézout theorem we have that $E_{i}^{ \pm} \cdot H=5$. Therefore, since $E_{i}^{ \pm} \cap H$ contains five points, we conclude that $E_{i}^{ \pm}$meets $H$ transversally in every point $P_{r}, r \in\{1,2,3,4,5\}$. It follows that $E_{i}^{ \pm}$meets $L_{1}$ transversally in every point $P_{r}, r \in\{1,2,3\}$. In conclusion

$$
E_{i}^{ \pm} \cdot L_{1}=1+1+1=3
$$

Therefore, for any $i \in\{0,1,2,3,4\}$ we have the following multiplicities:

$$
\begin{aligned}
& \eta\left(E_{i}^{+}\right) \cdot M_{1}=3, \\
& \eta\left(E_{i}^{-}\right) \cdot M_{1}=3 .
\end{aligned}
$$

Moreover, since the degree of $E_{i}^{ \pm}$is equal to 5 , it follows that $E_{i}^{ \pm} \cdot K_{Y}=5$, hence

$$
\begin{aligned}
& \eta\left(E_{i}^{+}\right) \cdot K_{X}=1, \\
& \eta\left(E_{i}^{-}\right) \cdot K_{X}=1 .
\end{aligned}
$$


Now we see that

$$
\Phi \cdot \eta\left(E_{i}^{ \pm}\right)=\left(3 K_{X}-M_{1}\right) \cdot \eta\left(E_{i}^{ \pm}\right)=3 K_{X} \cdot \eta\left(E_{i}^{ \pm}\right)-M_{1} \cdot \eta\left(E_{i}^{ \pm}\right)=3 \cdot 1-3=0 .
$$

Therefore the curves $\eta\left(E_{i}^{ \pm}\right)$do not intersect general fiber of the pencil $\Phi: X \rightarrow \mathbb{P}^{1}$. This proves the claim.

Consequently, any elliptic curve $\eta\left(E_{i}^{ \pm}\right)$lies in some reducible fiber $F_{i}^{+} \cup F_{i}^{-}$, i.e. $\eta\left(E_{i}^{ \pm}\right)=F_{i}^{ \pm}$. Moreover from the above computations it follows that $F_{i}^{ \pm} \cdot M_{1}=3$ and $F_{i}^{ \pm} \cdot K_{X}=1$. By the results in $[\mathrm{Re}]$ we also have : $F_{i}^{+} \cdot F_{i}^{-}=1, F_{i}^{+} \cdot F_{j}^{+}=0$ and $F_{i}^{-} \cdot F_{j}^{-}=0$ for $i \neq j$.

The multiplicities $F_{i}^{ \pm} \cdot M_{2}$ and $F_{i}^{ \pm} \cdot M_{3}$ can be computetd in a similar way using the explicite equations of the curves $E_{i}^{ \pm}$.

Therefore the intersection matrices of the 9 divisors $M_{1}, M_{2}, M_{3}, K_{X}, F_{0}^{+}, F_{1}^{+}, F_{2}^{+}, F_{3}^{+}$ and $F_{4}^{+}$on $X$ looks as follows

\begin{tabular}{|c|c|c|c|c|c|c|c|c|c|}
\hline & $M_{1}$ & $M_{2}$ & $M_{3}$ & $K_{X}$ & $F_{0}^{+}$ & $F_{1}^{+}$ & $F_{2}^{+}$ & $F_{3}^{+}$ & $F_{4}^{+}$ \\
\hline$M_{1}$ & -3 & 1 & 1 & 1 & 3 & 3 & 3 & 3 & 3 \\
\hline$M_{2}$ & 1 & -3 & 1 & 1 & 1 & 0 & 2 & 2 & 0 \\
\hline$M_{3}$ & 1 & 1 & -3 & 1 & 1 & 2 & 0 & 0 & 2 \\
\hline$K_{X}$ & 1 & 1 & 1 & 1 & 1 & 1 & 1 & 1 & 1 \\
\hline$F_{0}^{+}$ & 3 & 1 & 1 & 1 & -1 & 0 & 0 & 0 & 0 \\
\hline$F_{1}^{+}$ & 3 & 0 & 2 & 1 & 0 & -1 & 0 & 0 & 0 \\
\hline$F_{2}^{+}$ & 3 & 2 & 0 & 1 & 0 & 0 & -1 & 0 & 0 \\
\hline$F_{3}^{+}$ & 3 & 2 & 0 & 1 & 0 & 0 & 0 & -1 & 0 \\
\hline$F_{4}^{+}$ & 3 & 0 & 2 & 1 & 0 & 0 & 0 & 0 & -1 \\
\hline
\end{tabular}

Similarly for $M_{1}, M_{2}, M_{3}, K_{X}, F_{0}^{-}, F_{1}^{-}, F_{2}^{-}, F_{3}^{-}$and $F_{4}^{-}$one gets:

\begin{tabular}{|c|c|c|c|c|c|c|c|c|c|}
\hline & $M_{1}$ & $M_{2}$ & $M_{3}$ & $K_{X}$ & $F_{0}^{-}$ & $F_{1}^{-}$ & $F_{2}^{-}$ & $F_{3}^{-}$ & $F_{4}^{-}$ \\
\hline$M_{1}$ & -3 & 1 & 1 & 1 & 3 & 3 & 3 & 3 & 3 \\
\hline$M_{2}$ & 1 & -3 & 1 & 1 & 1 & 2 & 0 & 0 & 2 \\
\hline$M_{3}$ & 1 & 1 & -3 & 1 & 1 & 0 & 2 & 2 & 0 \\
\hline$K_{X}$ & 1 & 1 & 1 & 1 & 1 & 1 & 1 & 1 & 1 \\
\hline$F_{0}^{-}$ & 3 & 1 & 1 & 1 & -1 & 0 & 0 & 0 & 0 \\
\hline$F_{1}^{-}$ & 3 & 2 & 0 & 1 & 0 & -1 & 0 & 0 & 0 \\
\hline$F_{2}^{-}$ & 3 & 0 & 2 & 1 & 0 & 0 & -1 & 0 & 0 \\
\hline$F_{3}^{-}$ & 3 & 0 & 2 & 1 & 0 & 0 & 0 & -1 & 0 \\
\hline$F_{4}^{-}$ & 3 & 2 & 0 & 1 & 0 & 0 & 0 & 0 & -1 \\
\hline
\end{tabular}

The determinants of both intersection matrices are equal to -64 . Hence the cohomology classes of both collections of divisors in are linearly independent which proves the theorem

Now we recall some known facts about an involution on the Godeaux surface. Let the dihedral group $D_{10}$ act on $\mathbb{P}^{3}$ by adjoining to the action of a generator $\alpha$ of $\mu_{5}$ an involution $\beta$ defined by: $\beta: x_{i} \mapsto x_{5-i}$. If $Y$ is the Fermat quintic then $D_{10}$ acts on $Y$ and $\beta$ induces an involution (which we will again call $\beta$ ) on the Godeaux surface $X=Y / \mu_{5}$. It is easy to see that the three lines $L_{1}$, $L_{2}$ and $L_{3}$ are $\beta$-invariant. Therefore, $M_{1}, M_{2}$ and $M_{3}$ are $\beta$-invariant as well. Of course, the canonical class $K_{X}$ is invariant under $\beta$. Moreover, the explicit equations of the curves $E_{i}^{ \pm}$show that all curves $F_{i}^{ \pm}$are $\beta$-invariant. Hence we obtain the following

Proposition 2.2 Both algebraic basises for the $\mathbb{Q}$-vector space $A^{1}(X)=H^{2}(X, \mathbb{Q})$ appearing in Theorem 2.1 are invariant under the involution $\beta$. 
Now let $S=X / \beta$ be the quotient surface of the Godeaux surface $X$ by the involution $\beta$ and let

$$
\theta: X \rightarrow S
$$

be the corresponding quotient map. The involution $\beta$ on $X$ has five isolated fixed points $Q_{i}=$ $\eta\left(1:-\epsilon^{i}:-\epsilon^{i}: 1\right)$ and a fixed curve $M_{1}=\eta\left(L_{1}\right)$ with $M_{1}^{2}=-3$ and $M_{1} \simeq \mathbb{P}^{1}$. The quotient surface $S$ is a rational surface with five singular points $\theta\left(Q_{i}\right)$ (see [Re], p. 314). They are of type $(2,1)$ and locally isomorphic to the cone $z^{2}=x y$ (see [BPV], Th. 5.1, p. $81-82$, and Prop. 5.3, p. 84 - 85). Hence they can be resolved with a blow up $\mu: \tilde{S} \rightarrow S$ having an exceptional -2-curve, i.e. a curve $C \cong \mathbb{P}^{1}$ with selfintersection $C \cdot C=-2$. Let $\lambda: \tilde{X} \rightarrow X$ be the blow up of the surface $X$ at the 5 points $Q_{i}, i \in\{0, \ldots, 4\}$. Since $Q_{i}$ are smooth points, all exceptional fibers over $Q_{i}$ are -1 -curves. There exists a unique regular map $\tilde{\theta}: \tilde{X} \rightarrow \tilde{S}$, such that the diagram

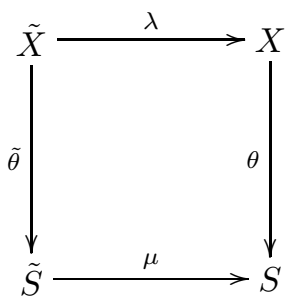

is commutative.

Corollary 2.3 For any $s \in\{0,1,2,3,4\}$ we have that $b_{s}(\tilde{X})=b_{s}(\tilde{S})$.

Proof. The surface $\tilde{X}$ is birationally isomorphic to the surface $X$. Hence $p_{g}(\tilde{X})=p_{g}(X)=0$ and $q(\tilde{X})=q(X)=0$ : therefore, $b_{1}(\tilde{X})=b_{1}(X)=0$ and, by Poincaré duality, $b_{3}(\tilde{X})=0$. Analogously, since $\tilde{S}$ is birationally isomorphic to $S$, we have that $q(\tilde{S})=q(S)=0$ and hence $b_{1}(\tilde{S})=b_{3}(\tilde{S})=0$. Clear that $b_{0}(\tilde{X})=b_{4}(\tilde{X})=1$ and $b_{0}(\tilde{S})=b_{4}(\tilde{S})=1$. Since $p_{g}=q=0$ for $\tilde{X}$ and for $\tilde{S}$, the two cycle maps $c l_{\tilde{X}}^{1}: A^{1}(\tilde{X}) \rightarrow H^{2}(\tilde{X}, \mathbb{Q})$ and $c l_{\tilde{S}}^{1}: A^{1}(\tilde{S}) \rightarrow H^{2}(\tilde{S}, \mathbb{Q})$ are both isomorphisms. Therefore, $b_{2}(\tilde{X})=b_{2}(\tilde{S})$ if and only if $\operatorname{dim}_{\mathbb{Q}} A^{1}(\tilde{X})=\operatorname{dim}_{\mathbb{Q}} A^{1}(\tilde{S})$.

Now let $Z$ be a projective surface, not necessarly smooth. From [Ful], 6.7 (e), it follows that, if $\tilde{Z} \rightarrow Z$ is the blow up of $Z$ in a finite set of $s$ points then $\operatorname{dim}_{\mathbb{Q}} A^{1}(\tilde{Z})=\operatorname{dim}_{\mathbb{Q}} A^{1}(Z)+s$. By taking $Z=X$ and $Z=S$ we get: $\operatorname{dim}_{\mathbb{Q}} A^{1}(\tilde{X})=\operatorname{dim}_{\mathbb{Q}} A^{1}(X)+5$ and $\operatorname{dim}_{\mathbb{Q}} A^{1}(\tilde{S})=\operatorname{dim}_{\mathbb{Q}} A^{1}(S)+5$. The homomorphism $\theta^{*}: A^{1}(S) \rightarrow A^{1}(X)$ is an embedding with image $A^{1}(X)^{\beta}=\left\{a \in A^{1}(X) \mid \beta(a)=\right.$ $a\}$. From Proposition 2.2 it follows that the $\mathbb{Q}$-vector space $A^{1}(X)$ has a $\beta$-invariant algebraic basis. Therefore $A^{1}(X)^{\beta}=A^{1}(X)$ which implies that $\operatorname{dim}_{\mathbb{Q}} A^{1}(S)=\operatorname{dim}_{\mathbb{Q}} A^{1}(X)=9$. Hence $\operatorname{dim}_{\mathbb{Q}} A^{1}(\tilde{S})=\operatorname{dim}_{\mathbb{Q}} A^{1}(\tilde{X})=9+5=14$. This shows that: $b_{2}(\tilde{S})=b_{2}(\tilde{X})=14$.

\section{$3 \quad$ Finite dimensional Motives}

Let $M=(X, p)$ and $N=(Y, q)$ be motives. The direct sum $M \oplus M$ is the motive $(X \amalg Y, p+q)$ where $X \amalg Y$ is the disjoint union of the two varieties and $p+q \in A^{0}(X, X) \oplus A^{0}(Y, Y)$.

The tensor product $M \otimes N$ is then defined to be the motive $(X \times Y, p \otimes q)$ where $X \times Y$ is the fiber product of the varieties and the tensor product of correspondences $p \otimes q$ is the intersection product $\pi_{X}^{*}(p) \cdot \pi_{Y}^{*}(q)$ where $\pi_{X}:(X \times Y) \times(X \times Y) \rightarrow X \times X$ and $\pi_{Y}:(X \times Y) \times(X \times Y) \rightarrow Y \times Y$. In particular this gives: $h(X) \otimes h(Y)=h(X \times Y)$.

For any variety $X$ let $X^{n}$ be $n$-times fiber product $X \times \cdots \times X$ over the base field $k$. If $f_{i}: X \rightarrow Y, i=1, \cdots, n$, are correspondences, that is $f_{i} \in A^{*}(X \times Y)$, then its tensor product 
$f_{1} \otimes \ldots \otimes f_{n}$ can be defined as follows. Let $p_{i}: X^{n} \times Y^{n} \rightarrow X \times Y$ be the projection defined by the rule $\left(\left(x_{1}, \cdots, x_{n}\right),\left(y_{1}, \cdots, y_{n}\right)\right) \mapsto\left(x_{i}, y_{i}\right)$. Then $f_{1} \otimes \ldots \otimes f_{n}$ is the intersection of correspondences $p_{1}^{*}\left(f_{1}\right) \cdot \ldots \cdot p_{s}^{*}\left(f_{s}\right)$. For a correspondence $f: X \rightarrow Y$ by $f^{(n)}$ denote its $n$-times tensor power $f \otimes \cdots \otimes f$. Then $f^{(n)} \in A^{*}\left(X^{n} \times X^{n}\right)$ : we will use $f^{(n)}$ instead of $f^{n}$ because the last symbol means the power of $f$ in the sense of compositions of correspondences, i.e. as en element of $A^{*}(X \times Y)$.

If $M$ is a motive by $M^{n}$ we will denote the $n$-th fold tensor product of $M$, that is $M^{n}=$ $M \otimes \ldots \otimes M$. A morphism $f: M \rightarrow N$ between motives is called smash nilpotent if $f^{(n)}=0$ for some $n>0$. One can also define exterior and symmetric powers of a motive $M$ in the following way. Let $\Sigma_{n}$ be the symmetric group of order $n$ : then there is a one-to-one correspondence between all irreducible representations of the group $\Sigma_{n}$ (over $\mathbb{Q}$ ) and all partitions of the integer $n$, where a partiton $n$ is an ordered collection of integers $\lambda_{1} \geq \ldots \geq \lambda_{s} \geq 0$, such that $\sum_{i=1}^{s} \lambda_{i}=n$.

Let $V_{\lambda}$ be the irreducible representation corresponding to a partition $\lambda$ of $n$ and let $\chi_{\lambda}$ be the character of the representation $V_{\lambda}$. Let

$$
d_{\lambda}=\frac{\operatorname{dim}\left(V_{\lambda}\right)}{n !} \sum_{\sigma \in \Sigma_{n}} \chi_{\lambda}(\sigma) \cdot \Gamma_{\sigma}
$$

where $\Gamma_{\sigma}$ is the correspondence $\left(x_{1}, \ldots, x_{n}\right) \mapsto\left(x_{\sigma(1)}, \ldots, x_{\sigma(n)}\right)$.

$\left\{d_{\lambda}\right\}$ is a set of pairwise orthogonal idempotents in $\operatorname{End}_{\mathcal{M}}\left(h\left(X^{n}\right)\right)$ such that $\sum d_{\lambda}=\Delta_{X^{n}}$. Hence they give a decompositon of the motive $h(X)^{n}=\left(X, \Delta_{X}\right)^{n}$ :

$$
h(X)^{n}=h\left(X^{n}\right)=\oplus_{\lambda}\left(X^{n}, d_{\lambda}\right) .
$$

If $M=(X, p)$ is a motive then $p^{(n)}$ is a projector on $X^{n}$ and $d_{\lambda} \circ p^{(n)}=p^{(n)} \circ d_{\lambda}$. Therefore the correspondences $p_{\lambda}^{(n)}=d_{\lambda} \circ p^{(n)}$ are pairwise orthogonal idempotents in $\operatorname{End}_{\mathcal{M}}\left(M^{n}\right)$, such that $\sum_{\lambda} p_{\lambda}^{(n)}=p^{(n)}$. In conclusion

$$
M^{n}=\left(X^{n}, p^{(n)}\right)=\oplus_{\lambda}\left(X^{n}, p_{\lambda}^{(n)}\right)
$$

where $\lambda$ runs over all partitions of $n$.

Definition 3.1 (see [Ki], 2.5) The $n$-th symmetric product of a motive $M=(X, p)$ is the motive $S^{n} M=\left(X, p_{\lambda}^{(n)}\right)$ where $\lambda$ is the partition $(n)$ and the $n$-th exterior power of $M$ is the motive $\wedge^{n} M=\left(X, p_{\lambda}^{(n)}\right)$ for $\lambda=(1, \ldots, 1)$. A motive $M$ is evenly finite dimensional if $\wedge^{n} M=0$ for some $n$, it is called oddly finite dimensional if $S^{n} M=0$ for some $n$.

We say that $M$ is finite dimensional if it can be decomposed as $M=M_{+} \oplus M_{-}$where $M_{+}$ is evenly finite dimensional and $M_{-}$is oddly finite dimensional. We also define $\operatorname{dim}\left(M_{+}\right)=m$ if $\wedge^{m} M_{+} \neq 0, \wedge^{m+1} M_{+}=0$ and similarly for $M_{-}$. For a finite dimensional motive $M$ we let $\operatorname{dim}(M)=\operatorname{dim}\left(M_{+}\right)+\operatorname{dim}\left(M_{-}\right)$.

The following criterium for a motive $M$ to be isomorphic to the motive of a point can be used to prove finite dimensionality in certain cases.

Proposition 3.2 ([Ki], 2.11) A motive $M=(X, p)$, with $d=\operatorname{dim}(X)$, is isomorphic to the motive of a point if there exist cycles $\alpha, \beta \in A^{*}(X)$ such that $p=\pi_{1}^{*}(\alpha) \cdot \pi_{2}^{*}(\beta)$ in $A^{d}(X \times X)$ where $\pi_{i}: X \times X \rightarrow X, i=1,2$, are the projections and $\pi_{i}^{*}: A^{d}(X) \rightarrow A^{d}(X \times X)$ the corresponding homomorphisms. 
Examples: 1) If $M$ is isomorphic to the motive of a point then it is evenly one dimensional. In fact the group $\Sigma_{2}$ acts trivially on $M \otimes M$, so $\wedge^{2} M=0$.

2) The motive $M=h\left(\mathbb{P}^{n}\right)$ is evenly $(n+1)$ dimensional because it is isomorphic to a direct sum of $(n+1)$ motives isomorphic to the motive of a point.

3) Let $C$ be a smooth projective curve of genus $g$ and let $p \in C$ be a point. Then we have:

$$
h(C)=h^{0}(C) \oplus h^{1}(C) \oplus h^{2}(C)
$$

where $h^{i}(C)=\left(C, \pi_{i}\right), \pi_{0}=[p \times C], \pi_{2}=[C \times p]$ and $\pi_{1}=\Delta_{C}-\pi_{0}-\pi_{2}$. By a result of Shermenev [She] (see also [Ki], 4.2) the motive $h^{1}(C)$ is oddly $2 g$-dimensional and each of the motives $h^{0}(C)$ and $h^{2}(C)$ is evenly finite dimensional, being isomorphic to the motive of a point by Prop. 3.2. Then the motive $h(C)$ is finite dimensional as a sum of finite dimensional motives (see Prop. 3.3 below).

Proposition 3.3 If the motives $M$ and $N$ are finite dimensional then $M \oplus N$ is finite dimensional.

Proof. Let $M=M_{+} \oplus M_{-}$and $N=N_{+} \oplus N_{-}$be decompositions of the finite dimensional motives $M$ and $N$, such that $\wedge^{s} M_{+}=0, \wedge^{s} N_{+}=0, S^{s} M_{-}=0$ and $S^{s} N_{-}=0$ for some integer $s$. Let $n$ be an integer, such that $n>2 s$. Then we have

$$
\wedge^{n}\left(M_{+} \oplus N_{+}\right) \cong \bigoplus_{a=0}^{n} \wedge^{a} M_{+} \otimes \wedge^{n-a} N_{+}
$$

and

$$
S^{n}\left(M_{-} \oplus N_{-}\right) \cong \bigoplus_{a=0}^{n} S^{a} M_{-} \otimes S^{n-a} N_{-} .
$$

Since $n>2 s$, it follows that either $a>s$ or $n-a>s$. Therefore each summand in the last two direct sums are zero. Hence $\wedge^{n}\left(M_{+} \oplus N_{+}\right)=0$ and $S^{n}\left(M_{-} \oplus N_{-}\right)=0$.

Using Young tableau and Young symmetralizers (see $[\mathrm{FH}]$ ) one can prove a similar result for the tensor product of motives. More precisely one has the following :

Proposition 3.4 Let $M$ and $N$ be evenly or oddly finite dimensional motives. When $M$ and $N$ have the same parity (both even or odd) then $M \otimes N$ is evenly finite dimensional; when $M$ and $N$ have a different parity, then $M \otimes N$ is oddly finite dimensional. In both cases we have $\operatorname{dim}(M \otimes N) \leq \operatorname{dim}(M) \cdot \operatorname{dim}(N)$.

Proof. See $[\mathrm{Ki}], 5.10$.

Corollary 3.5 As a consequence, if $M$ and $N$ are finite dimensional, then $M \otimes N$ is also finite dimensional.

Proof. Since $M$ and $N$ are finite dimensional, we have corresponding decompositions $M=M_{+} \oplus$ $M_{-}$and $N=N_{+} \oplus N_{-}$where $M_{+}$and $N_{+}$are evenly finite dimensional and $M_{-}$and $N_{-}$are oddly finite dimensional. Then $M \otimes N$ is a sum of products of evenly and oddly finite dimensional motives. By Prop. 3.4 and Prop. 3.3 the motive $M \otimes N$ is finite dimensional.

Proposition 3.6 Let $\tilde{X}$ be the blow up of a variety $X$ in subvariety $Y \subset X$ of a pure codimension $r$. If the Chow motives $h(Y)$ and $h(X)$ are finite dimensional then $h(\tilde{X})$ is finite dimensional. 
Proof. Let $\mathbb{L}=\left(\mathbb{P}^{1},\left[\mathbb{P}^{1} \times p t\right]\right)$ be the Lefschetz motive. Then

$$
h(\tilde{X}) \cong h(X) \oplus\left(\oplus_{i=1}^{r-1} h(Y) \otimes \mathbb{L}^{i}\right)
$$

(see $[\mathrm{Ma}])$. The motive $\mathbb{L}$ is finite dimensional by Proposition 3.2 and the motives $h(W)$ and $h(V)$ are finite dimensional by assumption. By Prop. 3.3 and Prop. $3.4 h(\tilde{X})$ is also finite dimensional.

The converse of 3.6 is also true, in the sense that if the Chow motive of $\tilde{X}$ is finite dimensional so it is $h(X)$. In order to prove this result we will show more generally, that if a motive $N$ is isomorphic to a submotive of a finite dimensional motive $M$ then $N$ is finite dimensional too (Prop. 3.11).

First we give a definition of surjective and injective morphisms of motives. It is equivalent to the definition given in [Ki], 6.5. by the Manin identity principle (see Theorem 3.9 below).

Definition 3.7 Let $f: M \rightarrow N$ be a morphism in $\mathcal{M}$. Then $f$ is a surjective morphism if there exists a morphism $g: N \rightarrow M$, such that $f \circ g=1_{N}$. Under these assumptions $g: N \rightarrow M$ is an injective morphism and $N$ is a submotive in $M$. If a morphism of Chow motives is at the same time injective and surjective it is an isomorphism in the category $\mathcal{M}$.

Proposition 3.8 Let $f: M \rightarrow N$ be a surjective morphism, where $M=(X, p)$ and $N=(Y, q)$. Then there exist projectors $t$ and $s$ on $X$, such that $(X, p)=(X, t) \oplus(X, s)$, where $(X, s)$ is a motive isomorphic to $N$. The projectors $s$ and $t$ are orthogonal, $i . \quad e$. $s t=0$ and $t s=0$, and $p=s+t$. Conversely, if such projectors $s$ and $t$ exist then $(X, p)=(X, t) \oplus(X, s)$. In particular we obtain a canonical projection $(X, p) \rightarrow(X, s)$.

Proof. By Definition 3.7 there exists a morphism $g: N \rightarrow M$ such that $f \circ g=1_{N}$. Let $s=g f$ (here and below we drop $\circ$ for short): then $s$ is a projector of $X$ because $s^{2}=g f g f=g f=s$. Since $f s=f g f=f$ and $q f=f, f$ is a morphism from $(X, s)$ into $(Y, q)$. Since $s g=g f g=g$ and $g q=g$, $g$ is a morphism from $(Y, q)$ into $(X, s)$. Since $p s=p g f=g f=s$ and $s^{2}=s, s$ is a morphism from $(X, s)$ into $(X, p)$. Moreover $s^{2}=s$ and $s p=g f p=g f=s$, so that $s$ is also a morphism from $(X, p)$ into $(X, s)$. We have: $g f=s=1_{(X, s)}$ and $f g=1_{(Y, q)}$ so that $f:(X, s) \rightarrow(Y, q)$ and $g:(Y, q) \rightarrow(X, s)$ yield an isomorphism between the motives $(X, s)$ and $(Y, q)$. Let $t=p-s$. Then $t^{2}=(p-s)(p-s)=p^{2}-s p-p s+s^{2}$ and $p^{2}=p, s^{2}=s, p s=s$ and $s p=s$ : so we get $t^{2}=p-2 s+s=p-s=t$, i.e. $t$ is a projector of $X$. The projectors $t$ and $s$ are pairwise orthogonal because $t s=(p-s) s=p s-s^{2}=s-s=0$ and $s t=s(p-s)=s p-s^{2}=s-s=0$. From the equalities $t+s=p$ and $p s=0=s p$ it follows that $(X, p)=(X, t) \oplus(X, s)$ and $t:(X, p) \rightarrow(X, t)$ is a projection of $(X, p)=(X, t) \oplus(X, s)$ on the first factor while $s:(X, p) \rightarrow(X, s)$ is a projection on the second factor. Therefore every surjective morphim $f: M=(X, p) \rightarrow N=(Y, q)$ induces a decomposition of $M$ into the direct sum of 2 factors such that $f$ is the projection onto one factor and $g$ the injection of one factor into the direct sum. The poof of the inverse statement is also easy.

Let $X$ and $Y$ be two varieties and let $f \in A^{*}(X \times Y)$ be a correspondence. Then $f$ induces a homomorphism

$$
f_{*}: A^{*}(X) \rightarrow A^{*}(Y)
$$

by the formula

$$
f_{*}(a)=p_{Y *}\left(f \cdot p_{X}^{*}(a)\right) .
$$


Here $p_{X}$ and $p_{Y}$ are projections of $X \times Y$ on $X$ and $Y$ respectively.

Let $M=(X, p)$ be a motive. Define $A^{*}(M)$ as the subgroup $p_{*}\left(A^{*}(X)\right) \subset A^{*}(X)$. Let $f: M \rightarrow N$ be a morphism in $\mathcal{M}$, where $N=(Y, q)$. Then, by definition, $f \in A^{*}(X \times Y)$ is a correspondence, such that $f \circ p=f=q \circ f$. Hence, $f_{*} p_{*}=f_{*}=q_{*} f_{*}$. It follows that $f_{*}\left(A^{*}(M)\right) \subset A^{*}(N)$. Therefore one can define a morphism $f_{*}: A^{*}(M) \rightarrow A^{*}(N)$.

Let $f$ be a morphism from $M=(X, p)$ into $N=(Y, q)$ and let $Z$ be a third variety. Consider the morphism $f \otimes \Delta_{Z}: M \otimes h(Z) \rightarrow N \otimes h(Z)$. It defines the homomorphism

$$
f_{Z}=\left(f \otimes \Delta_{Z}\right)_{*}: A^{*}(M \otimes h(Z)) \rightarrow A^{*}(N \otimes h(Z)) .
$$

If $a \in A^{*}(M \otimes h(Z)) \subset A^{*}(X \times Z)$ then $f_{Z}(a)=a \circ f^{t}$, where $f^{t}$ is the transpose of $f$.

The following is the well known Manin's identity principle:

Theorem 3.9 Let $f: M \rightarrow N$ be a morphism in $\mathcal{M}$. Then $f$ is a surjective (injective) morphism if and only if $f_{Z}$ is surjective (injective) homomorphism for any variety $Z$. In particular, $f$ is an isomorphism if and only if $f_{Z}$ is an isomorphism for any $Z$.

Proof. See [Sch], p.170.

Remark 3.10 Let $M=h(X)$ be the motive of a variety $X$ and $N$ the motive of a variety $Y$ : let $f: M \rightarrow N$ be induced by a proper surjective morphism between $X$ and $Y$. Then $f$ is surjective (see [Ki], Remark. 6.6).

The following proposition follows from 3.9 and from [Ki], Prop 6.9.

Proposition 3.11 Let $M$ and $N$ be two Chow motives. Suppose that there exists a surjective morphism $M \rightarrow N$ or, equivalently, an injective morphism $N \rightarrow M$. Then, if $M$ is finite dimensional $N$ is also finite dimensional.

Proof. Let $f: M \rightarrow N$ be a surjective morphism. It is clear that $\wedge^{n} M \stackrel{\wedge^{n} f}{\longrightarrow} \wedge^{n} N$ and $S^{n} M \stackrel{S^{n} f}{\longrightarrow}$ $S^{n} N$ are also surjective morphisms for any $n$. Hence, if $M$ is oddly or evenly finite dimensional then $N$ enjoys the same property. Let $M$ be finite dimensional, i.e. $M=M_{+} \oplus M_{-}$where $M_{+}$ is evenly finite dimensional and $M_{-}$is oddly finite dimensional: then it is enough to show that there exists a decomposition $N=N_{+} \oplus N_{-}$and two surjective morphisms $f_{+}: M_{+} \rightarrow N_{+}$and $f_{-}: M_{-} \rightarrow N_{-}$, such that $f=f_{+}+f_{-}$. This is proved in [Ki], Prop 6.9.

Corollary 3.12 Let $\tilde{X}$ be the blow up of a variety $X$ along a smooth subvariety $Y$. Assme that the motive $h(\tilde{X})$ is finite dimensional. Then $h(X)$ is finite dimensional.

Proof. From (4) it follows that $h(X)$ is a direct summand of $h(\tilde{X})$. By 3.11 it follows that $h(X)$ is finite dimensional.

The following theorem shows that finite dimensionality of the motive $h(X)$ is a birational invariant for a smooth projective surface $X$.

Theorem 3.13 Let $X, Y$ be smooth projective surfaces which are birationally equivalent. Assume $h(X)$ is finite dimensional. Then $h(Y)$ is finite dimensional. In particular: the Chow motive of a rational surface is finite dimensional. 
Proof. Let $T$ be a birational transformation between $X$ and $Y$. Then $T$ can be factored into a finite sequence of monoidal transormations (i.e. blow-ups of points) and their inverses. More precisely there exits a surface $Z$ and two birational morphisms $f: Z \rightarrow X$ and $g: Z \rightarrow Y$, such that: $T=g \circ f^{-1}, f$ and $g$ can be factored into a composition of finitely many blow-ups of points. Therefore by Prop. $3.6 h(Z)$ is finite dimensional. Similarly: $h(Y)$ is isomorphic to a submotive of the finite dimensional motive $h(Z)$. Hence by Corollary 3.12 it is finite dimensional. The last statement immediately follows for the finite dimendionality of the motive of $\mathbb{P}^{2}$.

Suppose now that $\mathcal{M}$ is the category of Chow motives over an algebraically closed field of characteristic 0 and let $H^{*}$ be a Weil cohomology theory for smooth projective varieties over $k$. We want to show that if a finite dimensional motive $M=(X, p)$ is homologically trivial, i.e. $c l_{X}(p)=0$ in $H^{*}(X)$, then $M=0$ in $\mathcal{M}$. We will need some notation and lemmas.

Let $\Sigma_{n}$ be the symmetric group of permutations on the set $\{1,2, \ldots, n\}$. Any permutation $g \in \Sigma_{n}$ decomposes into a set $o(g)$ of disjoint cycles which are called cycles. For any cycle $\sigma \in o(g)$ let $\operatorname{Supp}(\sigma)$ be the integers in $\{1,2, \ldots, n\}$ which $\sigma$ permutes and let $l(\sigma)$ be the length of the cycle $\sigma$.

For any variety $X$ and for any $n$ let $p_{i}: X^{n} \times X^{n} \rightarrow X \times X$ be the following projection: $\left(x_{1}, \ldots, x_{n}, x_{n}^{\prime}, \cdots, x_{n}^{\prime}\right) \rightarrow\left(x_{i}, x_{i}^{\prime}\right)$.

Lemma 3.14 Let $Z$ be a variety and let $l$ be a positive integer. Let $\sigma$ be a cycle of lenght $l(\sigma)=l$ in $\Sigma_{l}$. Let $f \in A^{*}(Z \times Z)$ be a correspondence. Then:

(i)

$$
p_{1 *}\left(\left(\Gamma_{\sigma} \circ f^{(l)}\right) \cdot p_{2}^{*}\left(\Delta_{Z}\right) \cdot \ldots \cdot p_{l}^{*}\left(\Delta_{Z}\right)\right)=f^{l} .
$$

(ii) if $f$ has codimension $\operatorname{dim}(Z)$ then the intersection $\left(\Gamma_{\sigma} \circ f^{(l)}\right) \cdot p_{1}^{*}\left(\Delta_{Z}\right) \cdot \ldots \cdot p_{l}^{*}\left(\Delta_{Z}\right)$ is a zero cycle on $Z^{l} \times Z^{l}$.

Proof. By definition, $f^{(l)}$ is the intersection $p_{1}^{*}(f) \cdot \ldots \cdot p_{l}^{*}(f)$ in $A^{*}\left(Z^{l} \times Z^{l}\right)$. Since $\Gamma_{\sigma}$ is a subvariety consisting of points of type $\left\{\left(x_{1}, \ldots, x_{l}, x_{\sigma(1)}, \ldots, x_{\sigma(l)}\right)\right\}$ in $X^{l} \times X^{l}$, it follows that the composition $\Gamma_{\sigma} \circ f^{(l)}$ differs from $f^{(l)}$ just by permuting factors. Then observe that if $Z_{1}, \ldots, Z_{m}$ are varieties and $f_{i} \in A^{*}\left(Z_{i} \times Z_{i+1}\right)$ correspondences where $i \in\{1, \ldots, m-1\}$ one has the following equality

$$
f_{m-1} \circ \ldots \circ f_{1}=r_{*}\left(r_{1}^{*}\left(f_{1}\right) \cdot \ldots \cdot r_{m-1}^{*}\left(f_{m-1}\right)\right),
$$

where $r_{i}: Z_{1} \times \ldots \times Z_{m} \rightarrow Z_{i} \times Z_{i+1}$ are the projection $\left(z_{1}, \ldots, z_{m}\right) \rightarrow\left(z_{i}, z_{i+1}\right)$ and $r: Z_{1} \times \ldots \times$ $Z_{m} \rightarrow Z_{1} \times Z_{m}$ sends $\left(z_{1}, \ldots, z_{m}\right)$ to $\left(z_{1}, z_{m}\right)$. Therefore, after eventually renumbering the factors and taking $f_{1}=f, f_{2}=\Delta_{X}$ etc., we get:

$$
p_{1 *}\left(\left(\Gamma_{\sigma} \circ f^{(l)}\right) \cdot p_{2}^{*}\left(\Delta_{Z}\right) \cdot \ldots \cdot p_{l}^{*}\left(\Delta_{Z}\right)\right)=f \circ \Delta_{Z} \circ f \circ \ldots \circ \Delta_{Z} \circ f=f \circ \ldots \circ f=f^{l} .
$$

Note that the degree of a power of the correspondence $f$ is equal to the number $l$ because $\sigma$ is a cycle of lenght $l(\sigma)=l$.

Suppose that $f$ has codimension $w$ in $Z \times Z$, where $w=\operatorname{dim}(Z)$. Then $\Gamma_{\sigma} \circ f^{(l)}$ has codimension $w l$. The cycle $p_{1}^{*}\left(\Delta_{Z}\right) \cdot \ldots \cdot p_{l}^{*}\left(\Delta_{Z}\right)$ has also codimension $w l$. Hence the intersection $\left(\Gamma_{\sigma} \circ f^{(l)}\right)$. $p_{1}^{*}\left(\Delta_{Z}\right) \cdot \ldots \cdot p_{l}^{*}\left(\Delta_{Z}\right)$ has codimension $2 l w=\operatorname{dim}\left(Z^{l} \times Z^{l}\right)$ and therefore it is a zero cycle on $Z^{l} \times Z^{l}$.

Lemma 3.15 Let $M=(X, p)$ be a motive and let $f: M \rightarrow M$ be an endomorphism of $M$, which, as a cycle on $X \times X$ has codimension equal to the dimension of $X$. Then we have: 
(i) if $M$ is oddly finite dimensional and $n>\operatorname{dim}(M)$ then:

$$
p_{1 *}\left(\left(d_{\lambda} \circ f^{(n)}\right) \cdot p_{2}^{*}\left(\Delta_{X}\right) \cdot \ldots \cdot p_{n}^{*}\left(\Delta_{X}\right)\right)=\sum_{g \in \Sigma_{n}} w_{g} f^{l\left(\tau_{1}(g)\right)}=0
$$

where: $\Sigma_{n}$ denotes the symmetric group, $\tau_{1}(g)$ is the cycle in the permutation $g$ containing 1 , $w_{g} \in \mathbb{Q}$ and $\lambda$ is the partition $(n)$;

(ii) if $M$ is evenly finite dimensional and $n>\operatorname{dim}(M)$ then:

$$
p_{1 *}\left(\left(d_{\lambda} \circ f^{(n)}\right) \cdot p_{2}^{*}\left(\Delta_{X}\right) \cdot \ldots \cdot p_{n}^{*}\left(\Delta_{X}\right)\right)=\sum_{g \in \Sigma_{n}} \operatorname{sgn}(g) w_{g} f^{l\left(\tau_{1}(g)\right)}=0
$$

where $\lambda$ is the partition $(1, \ldots, 1)$.

Proof. Let $n$ be an integer grater than $\operatorname{dim}(M)$. Let $\lambda$ be the partition $(n)$ if $M$ is oddly finite dimensional and $\lambda=(1, \ldots, 1)$ if $M$ is evenly finite dimensional. Since the projector $d_{\lambda}$, defined by (3), commutes with $p^{(n)}$ and $p \circ f=f$ it follows that

$$
p_{\lambda}^{(n)} \circ p_{\lambda}^{(n)}=\left(d_{\lambda} \circ p^{(n)}\right) \circ\left(d_{\lambda} \circ f^{(n)}\right)=d_{\lambda} \circ d_{\lambda} \circ(p \circ f)^{(n)}=d_{\lambda} \circ f^{(n)} .
$$

Therefore, $d_{\lambda} \circ f^{(n)}$ is a morphism from $h\left(X^{n}\right)$ to the motive $T_{\lambda} M$, where $T_{\lambda} M=\left(X, p_{\lambda}^{(n)}\right)$ is $S^{n} M$ if $M$ is oddly finite dimensional and $T_{\lambda} M=\wedge^{n} M$ if $M$ is evenly finite dimensional. In any case $T_{\lambda} M=0$. Therefore the correspondence $d_{\lambda} \circ f^{(n)}$ is equal to zero. Let $p_{i}: X^{n} \times X^{n} \rightarrow X \times X$ be the projection: $\left(x_{1}, \ldots, x_{n}, x_{1}^{\prime}, \ldots, x_{n}^{\prime}\right) \rightarrow\left(x_{i}, x_{i}^{\prime}\right)$. Then, since $d_{\lambda} \circ f^{(n)}=0$, we have:

$$
p_{1 *}\left(\left(d_{\lambda} \circ f^{(n)}\right) \cdot p_{2}^{*}\left(\Delta_{X}\right) \cdot \ldots \cdot p_{n}^{*}\left(\Delta_{X}\right)\right)=0 .
$$

If $\sigma \in o(g)$ let $p_{\sigma}: X^{n} \times X^{n} \rightarrow X^{l(\sigma)} \times X^{l(\sigma)}$ be the projection indexed by the integers $i_{1}, \ldots, i_{l(\sigma)}$ of $\operatorname{Supp}(\sigma)$, i.e.

$$
p_{\sigma}:\left(x_{1}, \ldots, x_{n}, x_{1}^{\prime}, \ldots, x_{n}^{\prime}\right) \rightarrow\left(x_{i_{1}}, \ldots, x_{i_{l(\sigma)}}, x_{i_{1}}^{\prime}, \ldots, x_{i_{l(\sigma)}}^{\prime}\right) .
$$

For any cycle $\sigma \in o(g)$ let $\Gamma_{\sigma}$ be the graph of corresponding map on $X^{l(\sigma)}$. Then the product $\Gamma_{g} \circ f^{(n)}$ decomposes as follows:

$$
\Gamma_{g} \circ f^{(n)}=\prod_{\sigma \in o(g)} p_{\sigma}^{*}\left(\Gamma_{\sigma} \circ f^{(l(\sigma))}\right),
$$

where the sign $\prod$ means intersection in the Chow ring.

From the equality above we get

$$
p_{1 *}\left(\left(\Gamma_{g} \circ f^{(n)}\right) \cdot p_{2}^{*}\left(\Delta_{X}\right) \cdot \ldots \cdot p_{n}^{*}\left(\Delta_{X}\right)\right)=p_{1 *}\left(\prod_{\sigma \in o(g)} p_{\sigma}^{*}\left(\Gamma_{\sigma} \circ f^{(l(\sigma))}\right) \cdot p_{2}^{*}\left(\Delta_{X}\right) \cdot \ldots \cdot p_{n}^{*}\left(\Delta_{X}\right)\right) .
$$

Now grouping the terms $p_{i}^{*}\left(\Delta_{X}\right)$ according to the cycles $\sigma$ in $o(g)$ we also have:

$$
p_{1 *}\left(\prod_{\sigma \in o(g)} p_{\sigma}^{*}\left(\Gamma_{\sigma} \circ f^{(l(\sigma))}\right) \cdot p_{\sigma}^{*}\left(\Delta_{X}^{(l(\sigma))}\right)\right)=p_{1 *}\left(\prod_{\sigma \in o(g)} p_{\sigma}^{*}\left(\left(\Gamma_{\sigma} \circ f^{(l(\sigma))}\right) \cdot \Delta_{X}^{(l(\sigma))}\right)\right) .
$$

Let $\tau_{1}(g)$ be the cycle in $o(g)$ such that $1 \in \operatorname{Supp}\left(\tau_{1}(g)\right)$. Since $f$ has codimension equal to the dimension of $X$ we see- using Lemma 3.14 (ii)- that if $\sigma \in o(g)$ and $1 \notin S u p p(\sigma)$, then $\left(\Gamma_{\sigma} \circ f^{(l(\sigma))}\right)$. $\Delta_{X}^{(l(\sigma))}$ is a zero-cycle on $X^{(l(\sigma))} \times X^{(l(\sigma))}$. 
Let $w_{\sigma}=\left(\Gamma_{\sigma} \circ f^{(l(\sigma))}\right) \cdot \Delta_{X}^{(l(\sigma))}$. By the projection formula we get, for every $\sigma \in o(g) \backslash\left\{\tau_{1}(g)\right\}$, i.e. for every $\sigma$, such that $1 \notin \operatorname{Supp}(\sigma)$ :

$$
\begin{gathered}
w_{\sigma}=\left(\Gamma_{\sigma} \circ f^{(l(\sigma))}\right) \cdot \Delta_{X}^{(l(\sigma))}=\left(\Gamma_{\sigma} \circ f^{(l(\sigma))}\right) \cdot p_{1}^{*}\left(\Delta_{X}\right) \cdot \ldots \cdot p_{l(\sigma)}^{*}\left(\Delta_{X}\right)= \\
=p_{1 *}\left(\left(\Gamma_{\sigma} \circ f^{(l(\sigma))}\right) \cdot p_{2}^{*}\left(\Delta_{X}\right) \cdot \ldots \cdot p_{l(\sigma)}^{*}\left(\Delta_{X}\right)\right) \cdot \Delta_{X}= \\
=f^{l(\sigma)} \cdot \Delta_{X} \in A^{2 d}(X \times X)=\mathbb{Q},
\end{gathered}
$$

where $d$ is the dimension of $X$. Hence $w_{\sigma}=\operatorname{deg}\left(f^{l(\sigma)} \cdot \Delta_{X}\right)$.

Let $w_{g}$ be the product of all $w_{\sigma}$ where $\sigma \in o(g) \backslash\left\{\tau_{1}(g)\right\}$. Then $w_{g} \in \mathbb{Q}$ and

$$
p_{1 *}\left(\left(\Gamma_{g} \circ f^{(n)}\right) \cdot p_{2}^{*}\left(\Delta_{X}\right) \cdot \ldots \cdot p_{n}^{*}\left(\Delta_{X}\right)\right)=p_{1 *}\left(w_{g} p_{\tau_{1}(g)}^{*}\left(\left(\Gamma_{\tau_{1}(g)} \circ f^{\left(l\left(\tau_{1}(g)\right)\right.}\right) \cdot \Delta_{X}^{\left(l\left(\tau_{1}(g)\right)\right)}\right) .\right.
$$

By Lemma 3.14 (i) the cycle $\left(\Gamma_{\tau_{1}(g)} \circ f^{\left(l\left(\tau_{1}(g)\right)\right.}\right) \cdot \Delta_{X}^{\left(l\left(\tau_{1}(g)\right)\right)}$ equals $f^{l\left(\tau_{1}(g)\right)}$.

So for any $g \in \Sigma_{n}$ we have

$$
p_{1 *}\left(\left(\Gamma_{g} \circ f^{(n)}\right) \cdot p_{2}^{*}\left(\Delta_{X}\right) \cdot \ldots \cdot p_{n}^{*}\left(\Delta_{X}\right)\right)=w_{g} f^{l\left(\tau_{1}(g)\right)}
$$

Using (5) we see that if $\lambda=(n)$ :

$$
p_{1 *}\left(\left(d_{\lambda} \circ f^{(n)}\right) \cdot p_{2}^{*}\left(\Delta_{X}\right) \cdot \ldots \cdot p_{n}^{*}\left(\Delta_{X}\right)\right)=\sum_{g \in \Sigma_{n}} w_{g} f^{l\left(\tau_{1}(g)\right)}=0 .
$$

Similarly, if $\lambda=(1, \ldots, 1)$, we get:

$$
p_{1 *}\left(\left(d_{\lambda} \circ f^{(n)}\right) \cdot p_{2}^{*}\left(\Delta_{X}\right) \cdot \ldots \cdot p_{n}^{*}\left(\Delta_{X}\right)\right)=\sum_{g \in \Sigma_{n}} \operatorname{sgn}(g) w_{g} f^{l\left(\tau_{1}(g)\right)} .
$$

The following result appears in $[\mathrm{Ki}], 7.2$ :

Theorem 3.16 Let $M=(X, p)$ be a finite dimensional motive. Let $f: M \rightarrow M$ be an endomorphism of $M$, which, as a cycle on $X \times X$, has codimension equal to the dimension of $X$. Assume that the cohomology class $c_{X \times X}(f)=0$ : then $f$ is nilpotent.

Proof. Assume $M$ is oddly finite dimensional. Then by Lemma 3.15 (i) we have

$$
\sum_{g \in \Sigma_{n}} w_{g} f^{l\left(\tau_{1}(g)\right)}=0
$$

where

$$
w_{g}=\prod_{\sigma} w_{\sigma}=\prod_{\sigma} f^{l(\sigma)} \cdot \Delta_{X} \in \mathbb{Q}
$$

and $\sigma$ runs over all cycles in $o(g) \backslash\left\{\tau_{1}(g)\right\}$. Let $\tilde{\Sigma}_{n}$ be the subset of $\Sigma_{n}$ consisting of all permutations $g$ which which are not a cycle, i.e. which can be decomposed into the product of at least two disjoint cycles. If $g \in \Sigma_{n} \backslash \tilde{\Sigma}_{n}$, then $o(g)$ consists of only one cycle $\tau_{1}(g)$ and $l\left(\tau_{1}(g)\right)=n$. The corresponding monomial in (6) is equal to $f^{n}$. If $g \in \tilde{\Sigma}_{n}$ then the corresponding monomial is equal to $w_{g} f^{l\left(\tau_{1}(g)\right)}$ and $l\left(\tau_{1}(g)\right)<n$.

So $(6)$ can be written as follows:

$$
\sum_{g \in \Sigma_{n} \backslash \tilde{\Sigma}_{n}} f^{n}+\sum_{g \in \tilde{\Sigma}_{n}} w_{g} f^{l\left(\tau_{1}(g)\right)}
$$


This shows that $f$ satisfies some polynomial whose highest degree term is $f^{n}$. To compute the coefficient $m$ of $f^{n}$ in (7) note that it comes from permutations $g$ with only one cycle of length $n$. The contribution for each $g$ is $(-1)^{n+1}$ in the even case and 1 in the odd case. Therefore, $m=(n-1) !(-1)^{n+1}$ in the even case and $m=(n-1) !$ in the odd case. In any case $m \neq 0$ so that $f$ satisfies a non trivial polynomial of degree $n$. Now, since $f$ homologically equivalent to zero, it follows that $f^{t}$ is homologically equivalent to zero for any positive integer $t$. Therefore, $f^{t}$ is numerically equivalent to zero. In particular,

$$
\operatorname{deg}\left(f^{t} \cdot \Delta_{X}\right)=0
$$

for any $t$. Therefore $w_{\sigma}=\operatorname{deg}\left(f^{l(\sigma)} \cdot \Delta_{X}\right)=0$ and from (7) we get $m f^{n}=0$. Since we work with coefficients in $\mathbb{Q}$, it follows that $f^{n}=0$ in $A^{*}(X \times X)$, i.e. $f$ is nilpotent in the ring $\operatorname{End}_{\mathcal{M}}(M)$.

If $M$ is evenly finite dimensional the proof is analogous (use Lemma 3.15 (ii)).

Let $M$ be a finite dimensional motive and let $M=M_{+} \oplus M_{-}$be a decomposition of $M$, such that $M_{+}$is evenly finite dimensional and $M_{-}$is oddly finite dimensional. Then $f: M \rightarrow M$ is a sum of four morphisms $f_{1}: M_{-} \rightarrow M_{-}, f_{2}: M_{-} \rightarrow M_{+}, f_{3}: M_{+} \rightarrow M_{-}$and $f_{4}: M_{+} \rightarrow M_{+}$. Since $f_{2}$ and $f_{3}$ are morphisms between finite dimensional motives with a different parity, we have that they are smash nilpotent and hence nilpotent (see Prop. 6.1 and Prop. 2.16 in [Ki]). The morphisms $f_{1}$ and $f_{4}$ are nilpotent by the previous arguments. So, $f$ is a sum of four nilpotent morphisms. Hence $f$ is nilpotent.

Corollary 3.17 Let $M=(X, p)$ be a Chow-motive. Assume that $M$ is finite dimensional. If $c l_{X \times X}(p)=0$ in $H^{*}(X \times X)$ then $M=0$. In other words, any homologically trivial finite dimensional motive is equal to zero in the category of Chow motives $\mathcal{M}$.

Proof. The correspondence $p$ is a nilpotent by Theorem 3.16. Since $p$ is an idempotent, we have that $p=0$.

The following theorem shows that Bloch's conjecture holds when $h(X)$ is finite dimensional.

Theorem 3.18 Let $X$ be a smooth projective surface over $\mathbb{C}$ with $p_{g}=0$. Assume that the Chow motive $M=h(X)$ is finite dimensional. Then the Albanese kernel $T(X)$ vanishes.

Proof. Let $\left[\Delta_{X}\right]=\pi_{0}+\pi_{1}+\pi_{2}+\pi_{3}+\pi_{4}$ be a Chow-Kunneth decomposition in $A^{2}(X \times X)$ ([Mu1]). Here $\pi_{0}=[p t \times X]$ and $\pi_{4}=[X \times p t]$ are the trivial parts, $\pi_{1}$ is the Picard projector, $\pi_{3}=\tilde{\pi}_{3}-\pi_{1} \circ \tilde{\pi}_{3}$ where $\tilde{\pi}_{3}$ is the Albanese projector, i.e. the transpose $\pi_{1}^{t}$ of $\pi_{1}$ (see Remark 6.5 in [Mu1]), and $\pi_{2}=\Delta-\pi_{0}-\pi_{1}-\pi_{3}-\pi_{4}$. Then the $\pi_{i}$ 's are orthogonal idempotents and the image of $\pi_{i}$ under the cycle class map in $H^{4}(X \times X, \mathbb{Q})$ is the Kunneth component $\Delta(4-i, i)$.

Let $\left\{\alpha_{1}, \ldots, \alpha_{s}\right\}$, where $s=b_{2}(X)$, be a basis of the $\mathbb{Q}$-vector space $H^{2}(X, \mathbb{Q})$ and $\left\{\tilde{\alpha}_{1}, \ldots, \tilde{\alpha}_{s}\right\}$ the dual basis by the cup product identifying $H^{2}(X)$ with $H_{2}(X)$. Then $\alpha_{i} \cup \tilde{\alpha}_{j}=0$ in $H^{4}(X, \mathbb{Q})$ for $i \neq j$ and $\sum q_{i}\left(\alpha_{i} \otimes \tilde{\alpha}_{i}\right)=\Delta(2,2)$ in $H^{4}(X \times X, \mathbb{Q})$ for some $q_{i} \in \mathbb{Q}$.

The map $A^{1}(X) \rightarrow H^{2}(X, \mathbb{Q})$ being surjective (because $p_{g}(X)=0$ ) there exist divisors $\left\{a_{i}\right\}$ and $\left\{\tilde{a}_{i}\right\}$ in $A^{1}(X)$ such that $\sum q_{i}\left(a_{i} \otimes \tilde{a}_{i}\right)=\Delta(2,2)$. Let $\sigma_{2}=\sum q_{i}\left(a_{i} \otimes \tilde{a}_{i}\right)$ : then we have $\pi_{0} \circ \sigma_{2}=\sigma_{2} \circ \pi_{0}=0$ and similarly $\pi_{4} \circ \sigma_{2}=\sigma_{2} \circ \pi_{4}=0$. Moreover, $\left(\sigma_{2}\right)_{*}(x)=0$ for every $x \in A^{2}(X)$.

Let

$$
f=\pi_{0}+\pi_{1}+\sigma_{2}+\pi_{3}+\pi_{4}
$$


Then $f$ is an endomorphism of the motive $M=h(X)=\left(X, \Delta_{X}\right)$ and $f-\Delta_{X}$ is homologically equivalent to 0 . By Theorem $3.16\left(f-\Delta_{X}\right)^{n}=0$ in $A^{2}(X \times X)$ for some integer $n . A^{2}(X \times X)$ is an associative ring with unit $\Delta_{X}$. Therefore we get:

$$
\left(f-\Delta_{X}\right)^{n}=\sum\left(\begin{array}{c}
n \\
i
\end{array}\right) f^{i}+(-1)^{n} \Delta_{X}=0 .
$$

Let $x \in A^{2}(X)$ : then according to [Mu1] we have: $\pi_{0}(x)=\pi_{1}(x)=0, \pi_{4}(x)=\operatorname{deg}(x)$ and $\operatorname{ker}\left(\pi_{3}\right)=T(X)=\operatorname{ker}\left(A_{0}(X) \rightarrow \operatorname{Alb}(X)\right)$ where $A_{0}(X)$ is the group of 0-cycles of degree zero. If $x \in A_{0}^{2}(X)$ then $f(x)=\pi_{3}(x)$ and $\left(f-\Delta_{X}\right)^{n}(x)=0$. Therefore we get:

$$
(-1)^{n+1} x=\sum\left(\begin{array}{c}
n \\
i
\end{array}\right)\left(\pi_{3}(x)\right)^{i} .
$$

This shows that $\operatorname{ker}\left(\pi_{3}\right)=T(X)=0$.

The following results yields a motivic proof of [BKL].

Corollary 3.19 Let $X$ be a complex surface with Kodaira dimension less than 2. If $p_{g}(X)=0$ then Bloch's conjecture holds for $X$.

Proof. By Theorem 3.18 it is enough to show that $h(X)$ is finite dimensional. By Theorem 3.13 the finite dimensionality is a birational invariant, so we can just look at the birational classification of surfaces with Kodaira dimension $\kappa<2$. If $\kappa<0$ then $X$ is either rational or birational to $C \times \mathcal{P}^{1}$ where $C$ is a smooth curve. So, in both cases $h(X)$ is finite dimensional. If $0 \leq \kappa<2$ then $X$ is elliptic: if $p_{g}=q=0$ then $X$ is an Enriques surface and $h(X)$ is isomorphic to the motive of a rational surface (see [Co]), hence by $3.13 h(X)$ is finite dimensional. If $p_{g}=0$ and $q=1$ then $X$ is hyperelliptic i.e. it is isomorphic to the quotient of the product of two elliptic curves by the action of a finite group. By Prop. 3.5 and by Remark 3.10 the motive $h(X)$ is again finite dimensional. This concludes the proof.

Lemma 3.20 Let $X$ and $Y$ be smooth projective varieties over a field $k$ and let $f: X \rightarrow Y$ be a morphism of finite degree; then $h(Y)$ is isomorphic to a submotive of $h(X)$

Proof. Let $f$ be of degree $d$ : then the following equality between correspondences hold (see [Ma], p.450)

$$
\Gamma_{f}^{t} \circ \Gamma_{f}=d \circ 1_{h(Y)} .
$$

Therefore the morphism $1 / d \cdot \Gamma_{f}: h(Y) \rightarrow h(X)$ is a right inverse to the morphism $\Gamma_{f}^{t}: h(X) \rightarrow$ $h(Y)$. By Prop. $3.8 h(Y)$ is isomorphic to a submotive of $h(X)$.

The next result (Theorem 3.21) shows that if $X$ is a smooth projective surface over $\mathbb{C}$, such that $p_{g}=q=0$ and the motive $h(X)$ is finite dimensional, then $h^{2}(X)$ is "trivial", i.e. it is isomorhic to a finite direct sum of copies of the Lefschetz motive $\mathbb{L}$. In order to prove this result we need to "enlarge" the category $\mathcal{M}$ of Chow effective motives over a field $k$ by adjoining the Tate twists $M(r)$ for every $M \in \mathrm{Ob}(\mathcal{M})$ and every $r \in \mathbb{Z}$. We then obtain a category $\overline{\mathcal{M}}$ whose objects are triples $M=(X, p, m)$ where $p$ is a projector and $m \in \mathbb{Z}$ (see [Sch], 1.4). Morphisms from $M=(X, p, m)$ into $N=(Y, q, n)$ in the category $\overline{\mathcal{M}}$ are given by correspondences $f \in A^{n-m}(X, Y)$ of degree $n-m$ such that $f \circ p=q \circ f=f$, where $A^{r}(X, Y)=A^{d+r}(X \times Y)$ if $X$ is purely $d$ dimensional. Clearly $\mathcal{M}$ is a full subcategory of $\overline{\mathcal{M}}$. The Lefschetz motive $\mathbb{L}$ is then isomorphic in $\overline{\mathcal{M}}$ to $(\operatorname{Spec}(k), \mathrm{id},-1)$. For every motive $M=(X, p, m)$ one defines the Tate twist $M(r)$ to be the 
motive $M \otimes \mathbb{L}^{-r}=(X, p, m+r)$, where $\mathbb{L}^{r}=\mathbb{L}^{\otimes^{r}}$ for a positive integer $r, \mathbb{L}^{0}=1$ and $\mathbb{L}^{r}=\mathbb{L}^{\otimes^{-r}}$ for a negative $r$ (see [Sch], 1.9).

$\overline{\mathcal{M}}$ is a pseudoabelian $\mathbb{Q}$-linear tensor category. It is then easy to see that all definitions and formal properties of finite dimensionality for $\mathcal{M}$ also hold in $\overline{\mathcal{M}}$ (or, more generally, in every pseudoabelian $\mathbb{Q}$-linear tensor category). In particular direct sums and tensor products of finite dimensional motives in $\overline{\mathcal{M}}$ are finite dimensional. If $f: M \rightarrow N$ is a surjective morphism in $\overline{\mathcal{M}}$ (or, equivalently, if $N \rightarrow M$ is injective) and if $M$ is finite dimensional then $N$ is finite dimensional too.

If $H^{*}$ is a Weil cohomology theory for smooth projective varieties over $k$ then one defines a functor $H^{i}$ on $\overline{\mathcal{M}}$, for every $i \in \mathbb{Z}$ by $H^{i}((X, p, m))=p_{*} H^{i+2 m}(X)$.

Theorem 3.21 Let $X$ be a smooth projective surface over $\mathbb{C}$, such that $p_{g}(X)=q(X)=0$. Assume that the motive $h(X)$ is finite dimensional. Then:

$$
h(X) \cong 1 \oplus b_{2}(X) \cdot \mathbb{L} \oplus \mathbb{L}^{2},
$$

where $b_{2}(X)=\operatorname{dim}\left(H^{2}(X, \mathbb{Q})\right)$.

Proof. By [Mu1] $X$ has a Chow-Kunneth decomposition $h(X)=\sum h^{i}(X)$. Since $H^{1}(X)=$ $H^{3}(X)=0$ we get $h(X)=h^{0}(X) \oplus h^{2}(X) \oplus h^{4}(X)$ where $h^{i}(X)=\left(X, \pi_{i}, 0\right)$ and $\Delta_{X}=\pi_{0}+\pi_{2}+\pi_{4}$. $h^{0}(X)$ and $h^{4}(X)$ are the trivial parts of $h(X)$ : in $\overline{\mathcal{M}}$ there are isomorphisms $h^{0}(X) \cong 1$ and $h^{4}(X) \cong \mathbb{L}^{2}$ (see [Sch], 1.13). By [Sch], 2.1 we have:

$$
\operatorname{Hom}_{\overline{\mathcal{M}}}\left(\mathbb{L}, h^{2}(X)\right)=\operatorname{Hom}_{\overline{\mathcal{M}}}\left((\operatorname{Spec}(\mathbb{C}), \mathrm{id},-1),\left(X, \pi_{2}, 0\right)\right)=\pi_{2} \circ A^{1}(\operatorname{Spec}(\mathbb{C}), X)=\pi_{2 *} A^{1}(X) .
$$

Since $\pi_{2}=\Delta_{X}-\pi_{0}-\pi_{4}$ and projectors $\pi_{0}$ and $\pi_{4}$ acts as zero on $A^{1}(X)$ (see [Mu1], Th. 3 ) we get $\left(\pi_{2}\right)_{*} A^{1}(X)=\left(\Delta_{X}\right)_{*} A^{1}(X)=A^{1}(X)$. Hence,

$$
\operatorname{Hom}_{\overline{\mathcal{M}}}\left(\mathbb{L}, h^{2}(X)\right)=\left(\pi_{2}\right)_{*} A^{1}(X)=A^{1}(X) .
$$

Analogously,

$$
\operatorname{Hom}_{\overline{\mathcal{M}}}\left(h^{2}(X), \mathbb{L}\right)=\operatorname{Hom}_{\overline{\mathcal{M}}}\left(\left(X, \pi_{2}, 0\right),(\operatorname{Spec}(\mathbb{C}), \mathrm{id},-1)\right)=A^{1}(X) .
$$

Note that $A^{1}(X) \cong H^{2}(X, \mathbb{Q})$, because $p_{g}(X)=q(X)=0$. Let $\alpha \in H^{2}(X, \mathbb{Q})$ : then $\alpha=$ $\sum q_{i}\left[e_{i}\right]$ where $\left[e_{i}\right]$ for $i=1, \ldots, b_{2}$ is a basis of the $\mathbb{Q}$-vector space $H^{2}(X, \mathbb{Q}) \cong A^{1}(X)$. Let $f_{\alpha}: \mathbb{L} \rightarrow h^{2}(X)$ be the corresponding morphism in $\overline{\mathcal{M}}$. Then $f_{\alpha}=\sum q_{i}\left[\operatorname{Spec}(\mathbb{C}) \times e_{i}\right]$. The transpose $f_{\alpha}^{t}$ is a morphism $h^{2}(X) \rightarrow \mathbb{L}([\mathrm{Sch}], 2.1)$ and

$$
f_{\alpha}^{t} \circ f_{\alpha}=\sum q_{i}[\operatorname{Spec}(\mathbb{C}) \times \operatorname{Spec}(\mathbb{C})] \in \operatorname{Hom}_{\overline{\mathcal{M}}}(\mathbb{L}, \mathbb{L}) \cong A^{0}(\operatorname{Spec}(\mathbb{C}) \times \operatorname{Spec}(\mathbb{C})) \cong \mathbb{Q} .
$$

Therefore, if we take $\alpha=e_{i}$ then the corresponding morphism $f_{i}=f_{e_{i}}: \mathbb{L} \rightarrow h^{2}(X)$ is injective. Let $f=\sum f_{i}: b_{2} \cdot \mathbb{L} \rightarrow h^{2}(X)$ : then $f$ is an injective morphism in $\overline{\mathcal{M}}$. By Proposition 3.8 (see also [Sch], Remark 1.7) there exists a motive $N=(Y, q, n)$ in $\overline{\mathcal{M}}$, such that $h^{2}(X) \cong b_{2} \cdot \mathbb{L} \oplus N$. $h(X)$ being finite dimensional, $h^{2}(X)$ is also finite dimensional. Hence $N$ is finite dimensional.

By taking cohomology we get:

$$
H^{i}\left(h^{2}(X)\right)=H^{i}\left(b_{2} \cdot \mathbb{L}\right) \oplus H^{i}(N) .
$$

By the results in [Mu1]: $H^{i}\left(h^{2}(X)\right)=0$ unless $i=2$ and $H^{2}\left(h^{2}(X)\right)=H^{2}(X, \mathbb{Q})$. Hence $\operatorname{dim}\left(H^{*}\left(h^{2}(X)\right)\right)=b_{2}(X)$. On the other hand: $H^{i}(\mathbb{L})=H^{i}((\operatorname{Spec}(\mathbb{C}), \mathrm{id},-1))=H^{i-2}(\operatorname{Spec}(\mathbb{C}))=$ 
0 unless $i=2$ in which case $H^{2}(\mathbb{L})=\mathbb{Q}$. Therefore $\operatorname{dim}\left(H^{*}\left(b_{2}(X) \cdot \mathbb{L}\right)\right)=b_{2}(X)$. It follows that $\operatorname{dim}\left(H^{*}(N)\right)=0$, that is $H^{i}(N)=0$ for all $i$. Let $n$ be an integer, such that $N(-n)$ lies in $\mathcal{M}$. Since $N$ is finite dimensional, $N(-n)$ is also finite dimensional, and since $H^{*}(N)=0$, it follows that $H^{*}(N(-n))=0$. Then, by Corollary 3.17, $N(-n)=0$. Therefore $N=0$ in the enlarged category $\overline{\mathcal{M}}$. Hence $h^{2}(X) \cong b_{2} \cdot \mathbb{L}$. This implies $h(X) \cong 1 \oplus b_{2} \cdot \mathbb{L} \oplus \mathbb{L}^{2}$.

\section{The Chow motive of the Godeaux surface}

Let $X$ be the Godeaux surface and let $\tilde{X}, \tilde{S}$ be defined as in $\S 2$. First we show, using the results in $\S 3$, that the Chow motives of the surfaces $X, \tilde{X}$ and $\tilde{S}$ are all finite dimensional.

Theorem 4.1 Let $Y$ be the Fermat quintic in $\mathbb{P}^{3}$, let $X=Y / \mu_{5}$ be the Godeaux surface. The Chow motive $h(X)$ is finite dimensional.

Proof. We will use the "inductive structure" on Fermat hypersurfaces proved in [Shi/Kat]: this result allows to construct a Fermat hypersurface from two Fermat hypersurfaces of smaller dimensions by blowing ups, taking quotients with respect to a cyclic group and blowing down.

It follows that the Fermat quintic $Y$ in $\mathbb{P}^{3}$ can be obtained from a product of two copies of the Fermat quintic plane curve $C$ defined by the equation $x_{1}^{5}+x_{2}^{5}+x_{3}^{5}=0$ in the following way. Let $c_{i}=\left(1:-\epsilon^{i}: 0\right) \in C$, where $0 \leq i \leq 4$ and $\epsilon$ is a generator of $\mu_{5}$. Let $Z$ be the blow up of the product $C \times C$ in the 25 points $c_{i} \times c_{j}$. Then $\mu_{5}$ acts on $Z$ and the quotient surface $Z / \mu_{5}$ is smooth (see Lemma 1.4 in [Shi/Kat]). Moreover the Fermat quintic $Y$ is obtained by blowing down two projective lines on $Z / \mu_{5}$ (see Lemma 1.5 and Lemma 1.6 in loc. cit.). The Chow-motive of a curve being finite dimensional (see $\S 3$ ) $h(C)$ is finite dimensional. By Proposition 3.4 the motive $h(C \times C)=h(C) \otimes h(C)$ is also finite dimensional. Since $Z$ is a blow up of $C \times C$ in a finite collection of points, the motive $h(Z)$ is finite dimensional by Proposition 3.6. The quotient map $Z \rightarrow Z / \mu_{5}$ induces a surjective morphism of Chow motives $h(Z) \rightarrow h\left(Z / \mu_{5}\right)$ (see Remark 3.10). Hence $h\left(Z / \mu_{5}\right)$ is finite dimensional by Proposition 3.11. By Corollary $3.12 h(Y)$ is finite dimensional too.

$h(Y)$ being finite dimensional, the motive $h(X)$ of the Godeaux surface $X$ is finite dimensional by Remark 3.10 and Proposition 3.11.

Corollary 4.2 Let $X$ be the Godeaux surface and let $\tilde{X}$ and $\tilde{S}$ be as in the end of Section 2. The Chow motives $h(\tilde{X})$ and $h(\tilde{S})$ are finite dimensional.

Proof. $\tilde{X}$ being the blow-up of $X$ at a finite number of points $h(\tilde{X})$ is finite dimensional by Proposition 3.6. The morphism $\tilde{\theta}: \tilde{X} \rightarrow \tilde{S}$ is a proper surjective map. Therefore $h(\tilde{S})$ is also finite dimensional.

Theorem 4.3 Let $X$ be the Godeaux surface. Then there exists an isomorphism of Chow motives:

$$
h(X) \cong 1 \oplus 9 \mathbb{L} \oplus \mathbb{L}^{2} .
$$

Let $\tilde{S}$ be a resolution of singularities of the rational surface $S=X / \beta, \beta$ being the involution on $X$. Then there exists an isomorphism of Chow motives:

$$
h(\tilde{S}) \cong h(X) \oplus 5 \mathbb{L}
$$

where $5 \mathbb{L}$ is a direct sum of five copies of $\mathbb{L}$. 
Proof. The first isomorphism immediately follows from Th. 3.21: in fact $h(X)$ is finite dimensional by 4.1 and $p_{g}(X)=q(X)=0, b_{2}(X)=9$.

To prove the second isomorphism we consider the same diagram as in Section 2 :

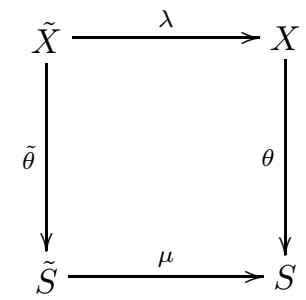

The map $\tilde{\theta}$ induces a morphism of motives $\Gamma_{\tilde{\theta}}: h(\tilde{S}) \rightarrow h(\tilde{X})$. Since $\theta: X \rightarrow S$ is a degree five map, the map $\tilde{\theta}$ has a degree five as well. Hence

$$
p=\frac{1}{5} \Gamma_{\tilde{\theta}} \circ \Gamma_{\tilde{\theta}}^{t}
$$

is a projector of the surface $\tilde{X}$ (see $[\mathrm{Ma}]$ ). Moreover,

$$
h(\tilde{X})=(\tilde{X}, p) \oplus\left(\tilde{X}, \Delta_{\tilde{X}}-p\right)
$$

and

$$
(\tilde{X}, p) \cong h(\tilde{S}) .
$$

In other words, the graph $\Gamma_{\tilde{\theta}}$ of the finite degree map $\tilde{\theta}$ embeds $h(\tilde{S})$ as a direct summand into the motive $h(\tilde{X})$. Let $M=\left(\tilde{X}, \Delta_{\tilde{X}}-p\right)$. Then $h(\tilde{X}) \cong h(\tilde{S}) \oplus M$. By Cor. $4.2 h(\tilde{X})$ is finite dimensional. By Proposition 3.11 the motive $M$ is also finite dimensional. The decomposition $h(\tilde{X})=h(\tilde{S}) \oplus M$ yields a similar the decomposition for the cohomology groups:

$$
H^{*}(h(\tilde{X})) \cong H^{*}(h(\tilde{S})) \oplus H^{*}(M)
$$

From Corollary 2.3 it follows that $b_{s}(\tilde{X})=b_{s}(\tilde{S})$ : hence $\operatorname{dim}\left(H^{*}(M)\right)=0$, i.e. $H^{*}(M, \mathbb{Q})=0$. From Corollary 3.17 we get $M=0$. Therefore, $h(\tilde{S})=h(\tilde{X})$. On the other hand, according to Manin's theorem (see $[\mathrm{Ma}], \S 9$ ), we have that $h(\tilde{X}) \cong h(X) \oplus 5 \mathbb{L}$. Therefore $h(\tilde{S}) \cong h(X) \oplus 5 \mathbb{L}$. This completes the proof of the theorem.

\section{References}

[BPV] W. Barth, C. Peters, A. Van de Ven. Compact Complex Surfaces. Ergebnisse der Mathematik und ihrer Grenzgebiete. 3 Folge. Band 4. Springer-Verlag, New-York, 1984 .

[Bl] S. Bloch. Lectures on algebraic cycles. Duke Univ. Math. Series IV, 1980.

[BKL] S. Bloch, A. Kas, D. Lieberman. Zero cycles on surfaces with $p_{g}=0$. Compositio Math. 33 (1976), 135 - 145.

[Co] K. Coombes. The K-cohomology of Enriques surfaces. Contemp. Math., 126 (1992) $47-57$.

[Ful] W. Fulton. Intersection theory. Ergebnisse der Mathematik und ihrer Grenzgebiete. 3 Folge. Band 2. Springer-Verlag, New-York, 1984. 
[FH] W. Fulton, J. Harris. Representation theory: a first course. Springer-Verlag, 1991.

[Ha] R. Hartshorne. Ample subvarieties of algebraic varieties. Lecture Notes in Math. 156 (1970).

[InMiz] H. Inose, M. Mizukami. Rational equivalence of zero-cycles on some surfaces with $p_{g}=0$. Math. Ann. 244 (1979) $205-217$.

[Ja] U. Jannsen, Motivic Sheaves and Filtratins on Chow Groups, In "Motives", Proc. Symposia in Pure Math. Vol. 55, Part 1 (1994), 245-302.

[Ki] S.-I. Kimura. Chow groups can be finite dimensional, in some sense. To appear in Journal of Algebraic Geometry.

[Kl] S. L. Kleiman. Algebraic cycles and the Weil conjectures. Dix Exposés sur la Cohomologie des Schémas. North-Holland, Amsterdam, 1968.

[Ma] Yu. I. Manin. Correspondences, motives and monoidal transformations. Math. USSR Sb. 6 (1968) 439 - 470.

[Mu1] J. P. Murre. On the motive of an algebraic surface. J. für die reine und angew. Math. Bd. 409 (1990), S. 190-204.

[Mu2] J. P. Murre. On a conjectural filtration on the Chow groups of an algebraic surface I, II. Indag. Math. 4 (2)(1990), pp.177-188, 189-201.

[Re] M. Reid. Campedelli versus Godeaux. In: Problems in the theory of surfaces and their classification (Cortona, 1988), pp. 309 - 365, Academic Press, 1991.

[She] A. M. Shermenev. The motive of an abelian variety. Funct. Anal. 8 (1974), 47 - 53.

[Sch] A. J. Scholl. Classical motives. In "Motives", Proc. Symposia in Pure Math. Vol.55, Part 1 (1994), pp.163-187.

[Shi/Kat] T. Shioda, T. Katsura. On Fermat varieties. Tôhoku Math. Journ. 31 (1979), 97 115 .

[Voi] C. Voisin. Sur les zero-cycles de certaine hypersurfaces munies d'un automorphisme. Ann. Scuola Norm. Sup. Pisa Ck. Si. (4) 19 (1992), 473 - 492. 